\title{
Modal Identification of a Centrifuge Soil Model Using Subspace State Space Method
}

\author{
H. Soltani ${ }^{\mathrm{a}}$, K. K. Muraleetharan ${ }^{\mathrm{a}}$, T. Runolfsson ${ }^{\mathrm{b}}$ \\ ${ }^{a}$ School of Civil Engineering and Environmental Science, University of Oklahoma, Norman, OK 73019, \\ United States.E-mail:soltani@ou.edu,muralee@ou.edu \\ ${ }^{b}$ School of Electrical and Computer Engineering, University of Oklahoma, Norman, OK 73019, \\ United States.E-mail: runolfsson@ou.edu
}

\begin{abstract}
In this paper, modal parameters of a layered soil system comprising of a soft clay layer overlying a dense sand layer are identified from accelerometer recordings in a centrifuge test. For the first time, the subspace state space system identification (4SID) method was employed to identify the natural frequencies, damping ratios, and complex valued mode shapes while considering the non-proportional damping in a soil system. A brief review of system identification concepts needed for application of the 4SID techniques to structural modal identification is provided in the paper. The identified natural frequencies were validated against those estimated by transfer function spectra. The computed normal mode shapes were compared with closed-form solutions obtained from the one-dimensional shear wave propagation equation. The identified modal parameters were then employed to synthesize state space prediction models which were subsequently used to simulate the soil response to three successive base motions. The identified models captured acceleration time-histories and corresponding Fourier spectra reasonably well in the small and moderate shaking events. In the stronger third shaking event, the model performed well at greater soil depths, but was less accurate near the surface where nonlinearities dominated.
\end{abstract}

Keywords: Subspace state space system identification (4SID); Natural frequencies, Mode shapes, Transfer function; Earthquake; Accelerometers; Centrifuge test; Layered soils

\section{Introduction}

Over the past 20 years, system theory-based structural identification has received increasing attention in experimental and operational modal analysis of instrumented bridges and buildings. Well-known state space oriented system identification methods include the Observer Kalman Filter [1,2], System Realization using the Information Matrix [3,4], Eigensystem Realization [5,6] and Subspace State Space System Identification (4SID) [2,6-10]. In most of these methods, the structural response recorded during vibrations induced by wind, traffic, earthquake and impact loads is employed to identify the associated state space model from which the modal parameters are extracted. An important consideration in geotechnical engineering is that the susceptibility of a structure to dynamic loads is determined not only by the modal characteristics of the structure itself, but also by the modal characteristics of the underlying soil structure [11-14].

In site response analysis, a great deal of effort has been invested to estimate the soil modal parameters from vertical accelerometer array data acquired in seismically active regions or in simplified models used in centrifuge and shake table tests [15-18]. The most widely used methods in this area include traditional correlation analysis, spectral analysis, and the shear beam method [19-22]. These techniques estimate the natural frequencies and damping coefficients using data from a pair of accelerometers, where both accelerometers may be embedded in the soil or one may be embedded with the other placed at the surface. Spectral analysis suffers from a number of potential issues such as leakage due to non-periodicity of the signal and sensitivity to the measurement noise. Moreover, with spectral analysis, a successful identification cannot be guaranteed unless wideband stationary signals are used for the excitations [23]. Consequently, alternative methods have been proposed to overcome these inherent problems of the frequency domain techniques. Glaser advocated the application of system identification techniques 
through implementing a variety of time invariant and time varying parametric models where the estimated model parameters were mapped to the mechanical parameters of a lumped-mass oscillator [24, 25]. These models proved surprisingly effective for predicting soil response to earthquakes: it was shown that some of the models extracted from the aftershock could predict the response during the main shock, which was unexpected since the main shock was characterized by significant nonlinearity. However, it should be noted that model order selection was difficult in these methods and only was achieved by empirically matching the model response to recorded data.

In this paper, we apply the 4SID method to identify the modal parameters of a soil system for the first time. To the best of our knowledge, this is the first time that soil system mode shapes have been identified while nonproportional damping in the soil system has been considered. We apply state space identification methods for soil dynamics and leverage their most attractive properties within a linear framework. While the resulting models are simple and intuitive, they prove in many cases to be accurate enough for the modeling objectives at hand and to provide improved understanding of the underlying physics of the system of interest. We consider a centrifuge soil model comprising of $10 \mathrm{~m}$ of soft clay over $8 \mathrm{~m}$ of dense sand. System identification is performed by applying the 4SID algorithm to acceleration-time histories recorded by a vertical array of five accelerometers and the recorded base motion. This procedure has many advantages. Chief among them is that it forms a basis for a rational choice of the optimal model size. Another interesting feature of this method is that the modal parameters of systems with general viscous damping can be identified, thereby providing a more realistic approximation of the damping mechanism in layered soils. This could prove significant in calibrating nonlinear ground response analysis models for damping at small strains as considered by Kwok et al., [26] and by Hashash and Park [27]. Our results can also be considered as an experimental validation of certain analytical solutions of the one dimensional viscoelastic wave propagation problem proposed for inhomogeneous soil deposits [28-31]. We briefly review the theoretical foundations of state space modeling as applied to general mechanical systems in Section 2. We then introduce the well-known 4SID algorithm that will be used in Section 3 to identify the modal parameters of the soil model. Theoretical validation of the identified modal parameters is also provided in Section 3. In Section 4, the state space models are synthesized using the identified parameters to predict the soil responses to three base motion events.

\section{Subspace state space identification of physical systems}

State space techniques for representing general mechanical systems are briefly reviewed in Section 2.1, while Section 2.2 presents the 4SID method that will be used in Section 3 to identify soil system modal parameters.

\subsection{State space representation of equation of motion in mechanical systems}

The coupled second-order differential equations for the motion of a mechanical system with $N$ degrees of freedom (DOFs) can be represented by a collection of first-order differential equations called state equations. By defining a physical state vector $x_{p}(t)=\left[\begin{array}{ll}d(t)^{T} & \dot{d}(t)^{T}\end{array}\right]^{T} \in \mathbb{R}^{2 N}$ containing the displacement $d(t) \in \mathbb{R}^{N}$ and velocity of all DOFs, the corresponding state space representation becomes

$\left[\begin{array}{cc}\boldsymbol{L} & \boldsymbol{M} \\ \boldsymbol{M} & \mathbf{0}\end{array}\right] \dot{x}_{p}(t)=\left[\begin{array}{cc}-\boldsymbol{K} & \mathbf{0} \\ \mathbf{0} & \boldsymbol{M}\end{array}\right] x_{p}(t)+\left[\begin{array}{l}\boldsymbol{\beta} \\ \mathbf{0}\end{array}\right] u(t)$

with mass matrix $\boldsymbol{M} \in \mathbb{R}^{N \times N}$, damping matrix $\boldsymbol{L} \in \mathbb{R}^{N \times N}$, and stiffness matrix $\boldsymbol{K} \in \mathbb{R}^{N \times N}$. The input influence matrix $\boldsymbol{\beta} \in \mathbb{R}^{N \times r}$ relates the excitation forces in vector $u(t) \in \mathbb{R}^{r}$ to the corresponding DOFs [32]. The response of the mechanical system $y(t)$ measured at $m$ DOFs can be expressed in terms of $m \times N$ displacement, velocity and acceleration output influence matrices $\boldsymbol{H}_{\boldsymbol{d}}, \boldsymbol{H}_{\boldsymbol{v}}$, and $\boldsymbol{H}_{\boldsymbol{a}}$ according to [32] as

$y(t)=\left[\begin{array}{lll}\boldsymbol{H}_{\boldsymbol{d}}-\boldsymbol{H}_{\boldsymbol{a}} \boldsymbol{M}^{-1} \boldsymbol{K} & \boldsymbol{H}_{\boldsymbol{v}}-\boldsymbol{H}_{\boldsymbol{a}} \boldsymbol{M}^{-\mathbf{1}} \boldsymbol{L}\end{array}\right] x_{p}(t)+\boldsymbol{H}_{\boldsymbol{a}} \boldsymbol{M}^{-1} \boldsymbol{\beta} u(t)$.

In cases, where only acceleration measurements are acquired, $\boldsymbol{H}_{\boldsymbol{d}}$ and $\boldsymbol{H}_{\boldsymbol{v}}$ become identically zero. The order of the state space model is defined as the number of state variables in the state vector, i.e. two times the number of DOFs. 
The modal parameters of the system can be obtained by solving the symmetric eigenvalue problem associated with Eq. (1). The eigenvalue problem can be diagonalized simultaneously if the eigenvectors are normalized with respect to the physical properties of the system i.e., $\boldsymbol{M}, \boldsymbol{L}$, and $\boldsymbol{K}$. The resulting diagonal matrices are given by

$\left[\begin{array}{c}\psi \\ \psi \Lambda\end{array}\right]^{\mathrm{T}}\left[\begin{array}{cc}L & M \\ M & 0\end{array}\right]\left[\begin{array}{c}\psi \\ \psi \Lambda\end{array}\right]=I$

$\left[\begin{array}{c}\psi \\ \psi \Lambda\end{array}\right]^{\mathrm{T}}\left[\begin{array}{cc}-K & 0 \\ 0 & M\end{array}\right]\left[\begin{array}{c}\psi \\ \psi \Lambda\end{array}\right]=\Lambda$

where $\boldsymbol{\Lambda} \in \mathbb{C}^{2 N \times 2 N}$ is a diagonal matrix with complex eigenvalues $\lambda_{i}$, and $\boldsymbol{V}=\left[\begin{array}{ll}\boldsymbol{\psi}^{T} & (\boldsymbol{\psi} \boldsymbol{\Lambda})^{T}\end{array}\right]^{T}$ is a $2 N \times 2 N$ matrix containing the corresponding complex eigenvectors $\psi_{i}, i=1 \ldots 2 N$. Other forms of scaling the eigenvectors are discussed in [33]. By changing the state vector to $\eta(t)$ given by $x_{p}(t)=\left[\begin{array}{ll}\boldsymbol{\psi}^{T} & (\boldsymbol{\psi} \boldsymbol{\Lambda})^{T}\end{array}\right]^{T} \eta(t)$ the system states are transferred to the modal space. The system equations in the modal form become

$\dot{\eta}(t)=\boldsymbol{\Lambda} \eta(t)+\boldsymbol{\psi}^{T} \boldsymbol{\beta} u(t)$,

$y(t)=\boldsymbol{H}_{\boldsymbol{a}} \boldsymbol{\Lambda}^{2} \boldsymbol{\psi} \eta(t)+\boldsymbol{H}_{\boldsymbol{a}} \boldsymbol{M}^{-\mathbf{1}} \boldsymbol{\beta} u(t)$,

where $\boldsymbol{H}_{\boldsymbol{a}}$ is an identity matrix for the case of acceleration measurements at all DOFs. The columns of $\boldsymbol{\psi}$ in Eqs. (5) and (6) are the complex displacement mode shapes. For under-damped systems, modes appear in complex conjugate pairs. A complex conjugate pair of eigenvalues accompanied by the corresponding complex conjugate pair of eigenvectors belong to one vibration mode. From the complex conjugate eigenvalue pairs the natural frequencies $f_{i}$ and damping ratios $\xi_{i}$ of each mode can be retrieved using the following equations [32]

$f_{i}=\frac{\left|\lambda_{i}\right|}{2 \pi}$,

$\xi_{i}=\frac{-R e\left(\lambda_{i}\right)}{\left|\lambda_{i}\right|}$.

The real-valued undamped or normal mode shape $\boldsymbol{\phi}$ can be extracted from the complex eigenvector $\boldsymbol{\psi}$ given in Eqs. (5) and (6) [32-34]. To this end, we assume that the external forces in $u(t)$ are imposed on the system at $r$ DOFs. However, in base excitation problems the external force at each DOF is modeled as a mass at that DOF multiplied by the base acceleration in the opposite direction. Thus, in Eq. (1), $\boldsymbol{\beta}$ becomes a vector containing the masses of all DOFs and $u(t)$ is simply the negative of the base acceleration. As a result, $y(t)$ in Eq. (6) represents the acceleration with respect to the base. In the next section, we will show how the state space model described in Eqs. (5) and (6) can be identified provided that the mass matrix, the base acceleration, and the structural response are available.

\subsection{Subspace state space system identification (4SID)}

In control and system theory, dynamic systems are modeled by differential or difference equations in the time domain. The system may be interpreted simply as a block that executes a prescribed set of mathematical operations on externally supplied inputs to calculate the outputs. The measured inputs $u(k)$ and outputs $y(k)$ of a discrete-time, linear, time-invariant, system can be fit into a discrete state space model

$x(k+1)=\boldsymbol{A}_{\boldsymbol{d}} x(k)+\boldsymbol{B}_{\boldsymbol{d}} u(k)$,

$y(k)=\boldsymbol{C}_{\boldsymbol{d}} x(k)+\boldsymbol{D}_{\boldsymbol{d}} u(k)$,

where $\boldsymbol{A}_{\boldsymbol{d}} \in \mathbb{R}^{n \times n}$ is the state matrix, $\boldsymbol{B}_{\boldsymbol{d}} \in \mathbb{R}^{n \times r}$ is the input matrix, $\boldsymbol{C}_{\boldsymbol{d}} \in \mathbb{R}^{m \times n}$ is the output matrix, $\boldsymbol{D}_{\boldsymbol{d}} \in \mathbb{R}^{n \times r}$ is a matrix through which inputs are directly fed into the outputs, and subscript $\boldsymbol{d}$ denotes discrete time. The state vector $x(k) \in \mathbb{R}^{n}$ includes $n$ state variables that describe the internal states of the system. The input is a 
deterministic vector $u(k) \in \mathbb{R}^{r}$ and $y(k) \in \mathbb{R}^{m}$ is the corresponding output vector. The goal of system identification is to determine the constant matrices $\boldsymbol{A}_{\boldsymbol{d}}, \boldsymbol{B}_{\boldsymbol{d}}, \boldsymbol{C}_{\boldsymbol{d}}$ and $\boldsymbol{D}_{\boldsymbol{d}}$ given $u(k)$ and $y(k)$ measured in an experiment. For state space identification of a controllable and observable system, a wide variety of methods are available [35]. In this paper we focus on 4SID, a relatively new technique which has been successfully employed recently in modal identification of a variety of complex structures including bridges [6-9]. Our objective in this section is not to provide a rigorous detailed treatment of 4SID, but rather to convey the basic ideas of the approach in an accessible way. Interested readers are referred to [35] for a comprehensive overview of data-driven or direct subspace identification methods and to [36] for a detailed treatment of 4SID.

Subspace state space methods begin by constructing the block Hankel matrices from the measured data samples. The past input and the past output block Hankel matrices are defined by

$\boldsymbol{U}_{0 \mid i-1}=\left[\begin{array}{cccc}u_{0} & u_{1} & \ldots & u_{j-1} \\ u_{1} & u_{2} & \ldots & u_{j} \\ \vdots & \vdots & \vdots & \vdots \\ u_{i-1} & u_{i} & \ldots & u_{i+j-2}\end{array}\right], \quad \boldsymbol{Y}_{0 \mid i-1}=\left[\begin{array}{cccc}y_{0} & y_{1} & \ldots & y_{j-1} \\ y_{1} & y_{2} & \ldots & y_{j} \\ \vdots & \vdots & \vdots & \vdots \\ y_{i-1} & y_{i} & \ldots & y_{i+j-2}\end{array}\right]$,

respectively. The block Hankel matrix of the future inputs $\boldsymbol{U}_{i \mid 2 i-1}$ and the future outputs $\boldsymbol{Y}_{i \mid 2 i-1}$ are formed in a similar way by adopting proper indices where the first number specifies the subscript of the entry in the first row of the first column while the second number specifies the subscript of the entry in last row of the first column. On the other hand, the subscript of the matrix entries identifies the data sample number. It is to be noted that each column is a time-shifted version of the previous column, and the distinction between the past and future can be assessed by checking the corresponding columns of $\boldsymbol{U}_{0 \mid i-1}$ and $\boldsymbol{U}_{i \mid 2 i-1}$ that they have no common entries. Here, $i$ is the number of block rows in the block Hankel matrices and is a user defined parameter. The value of this parameter is selected to be greater than the model order, but very smaller than the number of columns, $j$ [36]. The number of columns can be calculated known $i$ and the total number of time samples. By stacking the block Hankel matrices corresponding to the past data over the ones associated with the future data, $\boldsymbol{U}$ and $\boldsymbol{Y}$ matrices are constructed as

$\boldsymbol{U}=\left[\begin{array}{c}\boldsymbol{U}_{0 \mid i-1} \\ \boldsymbol{U}_{i \mid 2 i-1}\end{array}\right], \quad \boldsymbol{Y}=\left[\begin{array}{c}\boldsymbol{Y}_{0 \mid i-1} \\ \boldsymbol{Y}_{i \mid 2 i-1}\end{array}\right]$.

For consistent identification of a linear system it should be verified whether the input signal is persistently exciting. This condition is satisfied if the rank of $\boldsymbol{U}$ is equal to $2 \mathrm{ir}$ [36]. The performance of 4SID algorithm heavily relies on the assumptions imposed on the input data. A broadband and stationary signal with a high signal to noise ratio is ideal for a successful identification. Therefore, checking the input data for these assumptions is a crucial step.

System identification using subspace methods implements two approaches to estimate the matrices in the state space model defined by Eqs. (9) and (10): the shift invariance approach and the least-squares estimates of the system matrices provided that the state sequence $\boldsymbol{X}_{i}=\left[\begin{array}{llll}x_{i} & x_{i+1} & \cdots & x_{i+j-1}\end{array}\right]$ is known. Geometric operations of subspaces spanned by the column or row vectors of block Hankel matrices formed by the input and output data is used to estimate the state sequence. In this study we used the latter method where $\boldsymbol{Y}$ is decomposed into orthogonal matrices one of which is the product of the extended observabiliy matrix and the state sequence. Singular value decomposition (SVD) of the product of the extended observabiliy matrix and the state sequence determines the optimal order of the system. For an ideal noise-free data, the number of singular values greater than zero is the minimum model order whereas for noise-corrupted data with a low level of noise, the number of singular values above a noise threshold specifies the system order. Once the state sequence is estimated the system matrices $\widehat{A}_{\boldsymbol{d}}, \widehat{\boldsymbol{B}}_{\boldsymbol{d}}$, $\widehat{\boldsymbol{C}}_{\boldsymbol{d}}$, and $\widehat{\boldsymbol{D}}_{\boldsymbol{d}}$ can be extracted from

$\left[\begin{array}{cccc}x_{i+1} & x_{i+2} & \cdots & x_{i+j} \\ y_{i} & y_{i+1} & \cdots & y_{i+j-1}\end{array}\right]=\left[\begin{array}{cc}\widehat{\boldsymbol{A}}_{\boldsymbol{d}} & \widehat{\boldsymbol{B}}_{\boldsymbol{d}} \\ \widehat{\boldsymbol{C}}_{\boldsymbol{d}} & \widehat{\boldsymbol{D}}_{\boldsymbol{d}}\end{array}\right]\left[\begin{array}{llll}x_{i} & x_{i+1} & \cdots & x_{i+j-1} \\ u_{i} & u_{i+1} & \cdots & u_{i+j-1}\end{array}\right]$

When the number of data points $j \rightarrow \infty$ the optimization problem of determining the system matrices is solved in a least squares sense. A finite number of data points, presence of high noise level or over estimation of the system order might give rise to unstable models. For the detailed explanation of the identification algorithm and the geometric interpretation of the subspace method the readers are referred to [36,37]. 
The estimated system matrices can be transformed to their continuous time counterparts $\boldsymbol{A}_{\boldsymbol{c}}, \boldsymbol{B}_{\boldsymbol{c}}, \boldsymbol{C}_{\boldsymbol{c}}$ and $\boldsymbol{D}_{\boldsymbol{c}}$ using the zero-order hold assumption [38] in order to be consistent with the state space representation of the physical system in Eqs. (1) and (2). Note that $\boldsymbol{C}_{\boldsymbol{c}}=\widehat{\boldsymbol{C}}_{\boldsymbol{d}}$ and $\boldsymbol{D}_{\boldsymbol{c}}=\widehat{\boldsymbol{D}}_{\boldsymbol{d}}$. Using the eigenvectors of $\boldsymbol{A}_{\boldsymbol{c}}$, denoted by $\boldsymbol{\varphi}_{\boldsymbol{c}}$, the identified continuous time system can be converted to its modal coordinates $\mu(t)$ as defined by $x(t)=\boldsymbol{\varphi}_{c} \mu(t)$

$\dot{\mu}(t)=\Lambda_{c} \mu(t)+\varphi_{c}{ }^{-1} B_{c} u(t)$,

$y(t)=\boldsymbol{C}_{c} \boldsymbol{\varphi}_{c} \mu(t)+\boldsymbol{D}_{c} u(t)$,

where $\boldsymbol{\Lambda}_{\boldsymbol{c}}$ is a diagonal matrix containing eigenvalues of $\boldsymbol{A}_{\boldsymbol{c}}$. The eigenvalues of the continuous time model $\lambda_{c}$ are related to the eigenvalues of the discrete time model $\lambda_{d}$ through

$\lambda_{c}=\ln \left(\lambda_{d}\right) / T_{s}$.

where $T_{S}$ is the sampling interval. However, both the discrete and continuous time models have the same set of eigenvectors [38]. It worth mentioning that the eigenvectors of the state space model represented by Eqs. (5) and (6) are normalized with respect to the mass matrix. De Angelis and Imbimbo [39] showed that in base excitation problems, scaling the mode shapes can be performed having the mass of one DOF known a priori. The proposed scaling approach also demands a complete set of mode shapes in which the number of sensors or the elements in each mode shape vector is equal to the number of identified modes. For lumped-mass systems, this implies that all vibration modes are adequately excited and identified. Once the eigenvectors are scaled, the entire mass matrix as well as the stiffness and damping matrices of the system can be derived as $[33,40]$

$\boldsymbol{M}=\left(\boldsymbol{\psi} \boldsymbol{\Lambda} \boldsymbol{\psi}^{T}\right)^{-1}, \boldsymbol{L}=-\boldsymbol{M} \boldsymbol{\psi} \boldsymbol{\Lambda}^{2} \boldsymbol{\psi}^{T} \boldsymbol{M}, \boldsymbol{K}=-\left(\boldsymbol{\psi} \boldsymbol{\Lambda}^{-1} \boldsymbol{\psi}^{T}\right)^{-1}$.

\section{Modal identification of a layered soil model in a centrifuge test}

This section demonstrates implementation of 4SID algorithm for identifying the modal parameters of a soil deposit. It also shows how the identified parameters are validated and used to synthesize the predictive state space models.

\subsection{Test setup, instrumentation and data processing}

A centrifuge model at a scale of $1 / 30$ including seven single piles in a layered soil deposit was constructed to study the seismic behavior of single piles in improved and unimproved soft clays [41]. In this paper, we restrict our discussion to the free-field response of soil. All the results and dimensions in this paper are reported in the corresponding prototype scale. The unimproved soil profile consisted of a $10 \mathrm{~m}$ slightly overconsolidated soft clay layer overlying an $8 \mathrm{~m}$ of dense sand layers as shown in Fig. 1. The water table was above the soil surface. The sand layer consisted of dense Nevada sand $\left(\mathrm{D}_{50}=0.15 \mathrm{~mm}, \mathrm{e}_{\max }=0.887, \mathrm{e}_{\min }=0.511\right.$ and $\left.\mathrm{G}_{\mathrm{s}}=2.67\right)$ at an average void ratio of 0.54 . The soft clay $(\mathrm{LL}=32, \mathrm{PI}=15)$ layer consisted of equal portions of Kaolin and sand mixed at a water content of $64 \%$ and then consolidated in four layers at $1 \mathrm{~g}$ to various vertical stresses and subsequently reconsolidated in the centrifuge at $30 \mathrm{~g}$ to obtain a slightly overconsolidated clay layer. The model was then subjected to three successive earthquake motions with increasing level of intensity. As depicted in Fig. 2, the first and the second shaking events are the scaled 1989 Loma Prieta earthquake recorded in Santa Cruz with maximum accelerations of $0.12 \mathrm{~g}$ and $0.23 \mathrm{~g}$, respectively, while the third shaking event is the scaled 1995 Kobe earthquake with a peak acceleration of $0.84 \mathrm{~g}$. The applied excitations to the base of the model were oriented in the horizontal direction. Soil motion at five different depths was recorded by accelerometers A2, A6, A11, A16, and A26 placed at 2, 6, 9.3, 11.7 and $16.8 \mathrm{~m}$ from the base as marked with filled triangles in Fig. 1. The acceleration time histories were sampled at approximately $136 \mathrm{~Hz}$. In order to reduce the noise level induced by digitization, a fourth-order band-pass zero-phase Butterworth filter with low frequency $0.25 \mathrm{~Hz}$ and high frequency $7 \mathrm{~Hz}$ was applied to the recorded signals. It was assumed that the noise characteristics were inherent in the pre-event and post-event components of the data. Therefore, the cutoff frequencies of the filter were estimated by detecting the frequencies at 
which the Fourier spectrum of the signal in the main excitation started to diverge from that of the pre-event and post-event signals. It should be noted that filtering did not necessarily remove all the noise in the measurements.

\subsection{Identification of modal parameters and validation}

As the first step, the input data Hankel matrices $\boldsymbol{U}_{0 \mid i-1}$ and $\boldsymbol{U}_{i \mid 2 i-1}$ were constructed using the base acceleration recorded by A46 according to Eq. (12). In a similar way, the acceleration time histories of the embedded sensors (A2, A6, A11, A16, and A26) with respect to the base were used to form the Hankel matrices of the past and future output data, $\boldsymbol{Y}_{0 \mid i-1}$ and $\boldsymbol{Y}_{i \mid 2 i-1}$, respectively. Implementing 4SID requires two parameters to be determined a priori: the number of block rows $i$ and the appropriate model order $n$. Examining the rank of the input Hankle matrix $\boldsymbol{U}$ in Eq. (12) for different values of the block rows showed that row blocks higher than 12 would result in rank deficient $\boldsymbol{U}$ matrix; therefore, $i$ was selected as 12 . The performance of the state space identification methods depends greatly on how well the input excitation meets the underlying assumptions. When the excitation is narrowband and nonstationary, which is the case for earthquake excitations, the identified values depend on the length of the data used in the identification [1, 4]. Herein, different lengths of data were used in the identification. The number of columns $j$ depends on the number of selected data points contained in the Hankel matrices. In order to determine the model order, which corresponds to twice the number of structural modes, we started by inspecting the singular values of the product of the extended observabiliy matrix and the state sequence. From the singular values plot in Fig. 3 , it is clear that the number of significant singular values is not sensitive to the number of data points. These graphs show that except the fourth or fifth singular value, the rest are of very small values suggesting that two or three modes are present in the data.

In modal analysis, when trying to estimate the modal parameters from real data, especially in experimental modal analysis of large scale structures where the selection of the model order is difficult, it is a standard practice to over specify the model order above what is implied by the singular value plot [42]. This helps to detect the weakly excited system modes that appear only in systems with high orders. Although over specification helps to capture all the excited modes and reduces the bias in the estimated parameters, it creates a set of non-physical or mathematical modes due to the measurement noise or nonlinearities in the system. To distinguish between the true modes and the spurious modes, stabilization diagram were introduced which are the plots of modal parameters identified from the state space models with increasing order against the corresponding model order. These diagrams have been widely used in modal identification of bridges and shear-frame structures [1, 3, 6, 8, 10,37]. Not only the effect of the model order on the identified modal parameters is explored in these graphs, but also the effect of data length is considered as studied in $[1,4]$. In stabilization diagrams a true mode of the system lines up along the nearly same frequency as the model order increases. It also shows up with consistent damping and mode shapes. On the other hand, spurious modes are expected to show erratic behavior. These modes can be detected and removed by setting a validation criterion such that if the difference between the eigen frequencies of the two consecutive model order is larger than a pre-set value, these modes can be marked as spurious modes.

In developing the stabilization diagrams, discrete-time state space models with the model order changing from 2 to 12 (corresponding to systems with 1 to 6 modes) were identified while the model order was increased in steps of 2. The upper bound limit on the model order was imposed by the maximum number of rows in the Hankel matrix $\boldsymbol{U}$. In addition, the effect of data length was also studies by increasing the number of time samples introduced to the identification algorithm counted from the beginning of the excitation. The natural frequencies $f_{i}$ and damping ratios $\xi_{i}$ were extracted using Eqs. (7) and (8). As the number of time samples increased, the identified parameters became stabilized at certain frequency ranges as shown in Fig. 4. The number of stable modes in models with the order of 4 and 6 were so small that no stabilization diagram could be created. Therefore, only the parameters of the models with orders 8,10 and 12 are presented in the figure. The magnitude and phase of the associated complex mode shapes are depicted in Fig. 5. In order to improve the clarity of these diagrams, the unstable modes with negative damping ratios and modes with frequencies higher than $7 \mathrm{~Hz}$ (i.e. the upper cutoff frequency of the applied Butterworth filter) were excluded. It is a well known fact that constraints such as finite number of the samples/data points, modeling errors, plant and measurement noise can result in unstable modes and therefore unstable $\widehat{A}_{\boldsymbol{d}}$ matrix (Eq. (13)), even though the system being modeled in real world is stable. According to the definition [38] a discrete state space model is stable if and only if all the poles or the eigenvalues of matrix $\widehat{A}_{\boldsymbol{d}}$ lie inside the unit circle. To date, various methods have been proposed to force a stable identification of $\widehat{\boldsymbol{A}}_{\boldsymbol{d}}$ including zero-padding [43], data 
augmentation [44], regularization [45], and constrained optimization [46]. Some of these methods involve iterative process and bear a significant computational burden. Since the major objective of the current study is to construct predictive state space models using the identified modal parameters, the stabilizing methods have not been considered here.

The diagrams for natural frequencies in Figs. 4(b), (c) and (d) imply that three modes around $1 \mathrm{~Hz}, 3 \mathrm{~Hz}$ and in the frequency range of 5-6 Hz were excited. On the other hand, the stabilization diagrams for modal damping ratios show higher uncertainties in the estimated values. Difficulties in identification of modal damping ratios has been experienced and commonly reported in the structural identification literature [1, 3, 6, 8, 10,37]. In all these studies it was found that the uncertainty in estimation of damping ratios is high relative to the uncertainty in estimated natural frequencies. As stated in the aforementioned studies, small valued damping ratios are sensitive to non-stationary nature of seismic excitation and the presence of noise. This can partly explain the observed scatter in the damping ratio stabilization diagram. In order to facilitate easy interpretation of the stabilization diagrams of natural frequencies and damping ratios, modal parameters associated with a certain model order that varied between appropriately chosen threshold values as a result of increase in data length were grouped together. A total of 15 groups were detected including 3 groups of the identified vibration modes around $1 \mathrm{~Hz}, 7$ groups around $3 \mathrm{~Hz}$, and 5 groups in the frequency range of 5-6 Hz. From each group one representative complex mode shape was selected and used in the validation phase of this study as reported later in this section.

The identified natural frequencies were validated using the peaks in the transfer function spectra. The transfer functions were computed from the power spectral density (PSD) of the soil acceleration at a specific depth over the PSD of the base motion. Small frequency content of the input base motion at certain frequencies (Fig. 2(b)) caused spurious peaks in the transfer function spectra. Bartlett lag windows with lengths 700 were employed to remove these fictitious peaks where a total of 8800 data points were used in the spectral estimation. Computation of transfer functions involves substantial signal processing that can be found in [47-49]. The transfer function amplitude for each pair of base and soil acceleration data is shown in Fig. 6 (a). Two important observations can be made from this graph. First, the amplification in deeper layers occurs at frequencies greater than $2 \mathrm{~Hz}$, while in the uppermost layer amplification is pronounced at lower frequencies around $1 \mathrm{~Hz}$. This behavior is expected since soil layers act as lowpass filters removing the high frequencies in the propagating waves. As can be seen from this graph the identified natural frequencies are distributed around the peaks of the transfer functions showing a good agreement between the results of two different identification approaches, 4SID in the time domain and transfer function in the frequency domain. The transfer function for the pair A46-A2 and pair A46-A6 have values close to unity, indicating a negligible amplification of response within the dense sand layer. The above observation implies that the soil profile is likely responding as a single inhomogeneous layer where the response is dominated by the upper soft clay layer. As a side remark, the transfer function spectra obtained for the second shaking event (not shown here) showed noticeable changes compared to the first event reflecting the nonlinearity in the soil system.

The identified natural frequencies were also verified against the natural modes of an inhomogeneous viscoelastic soil layer over a rigid rock. Various research efforts on response of continuously inhomogeneous soils to vertically propagating shear waves have resulted in closed-form solutions for natural frequencies, mode shapes and base-tosurface amplification functions [28-31]. Exponential and power law variations of the shear wave velocity with depth are the special cases that simplify the solutions of the wave propagation differential equation in terms of Bessel functions or power series solutions. The shear wave velocity profile calculated from the cross-correlation of all combinations of two embedded accelerometers in the soil model is shown in Fig. 7(a). The shear wave velocity profile shows an exponentially increasing stiffness with depth $z$, which can be expressed by

$V_{s}(z)=V_{0} e^{a z}$.

Velocity at the soil surface $V_{0}$ and the inhomogeneity rate parameter $a$ were estimated using the least squares fit as $11.43 \mathrm{~m} / \mathrm{s}$ and 0.24 , respectively. The response of a viscoelastic soil layer to a harmonic motion with circular frequency $\omega$ in terms of horizontal displacement is governed by the following differential equation

$\frac{d}{d z}\left(G(z) \frac{d u}{d z}\right)+\rho \omega^{2} u=0$ 
where $G(z)$ is the continuously varying shear modulus with depth which can be obtained from the shear wave velocity profile knowing the soil mass density $\rho$. The solution of Eq. (19) is provided in [29] where it is assumed that the mass density of the soil is constant throughout the layers. A mass density of $973 \mathrm{~kg} / \mathrm{m}^{3}$ was estimated based on the weighted average of the mass density of the soil layers. The material damping is accounted for by replacing $G(z)$ by its complex counterpart as

$G(z) \rightarrow G(z)(1+2 \beta i)$

where $\beta$ is the linear hysteresis damping coefficient which was assumed as a constant for the entire soil profile. The natural frequencies are determined by assuming zero displacement at the interface of the soil and the bedrock. The natural frequencies of the soil model in the frequency range of interest $0-7 \mathrm{~Hz}$ were calculated as $1.08,2.48,3.90$, 5.34, $6.76 \mathrm{~Hz}$ implementing equation 2.134 in reference [29]. To investigate the impact of slight variations in the parameters of the exponential model in Eq. (18) on the estimated natural frequencies, a sensitivity analysis was conducted. The shear wave velocity estimated at the soil surface $V_{0}$ was varied from 12 to $20 \mathrm{~m} / \mathrm{s}$ while $a$ was changed accordingly to produce the best match with the original least-squares fit. The exponential model for the shear wave velocity profile is very sensitive to variations in parameter $a$. As a consequence, the fitted range selected as 0.21 to 0.245 was quite narrow. The results of this study showed that increasing $V_{0}$ shifts the natural frequencies towards higher frequencies while it spreads the same number of modes over a broader frequency band such that for the case with $V_{0}=20 \mathrm{~m} / \mathrm{s}$ only three modes with frequencies of $1.60,3.68$, and $5.79 \mathrm{~Hz}$ were situated within the frequency range of interest. Among the various cases considered in the sensitivity analysis, the case with $V_{0}=$ $14 \mathrm{~m} / \mathrm{s}$ and $a=0.235$ produced the closest match with the first and the second identified frequencies; therefore, the parameters of the shear wave velocity model were updated to $V_{0}=14 \mathrm{~m} / \mathrm{s}$ and $a=0.235$. Although representative of the experimental model in this study, the absolute value of $V_{0}$ can be considered quite low for real soft clays.

Equation 2.140 in reference [29] was devised to calculate the amplification function of the soil model as the ratio of the displacement at the soil surface to that of the base for damping coefficients $\beta=0.01,0.02,0.05$, and 0.1 as plotted in Fig. 6(b). In the analytical method, all modes possess the same damping ratio. Comparing the transfer functions in Fig. 6(a) with the amplification functions in Fig. 6(b) suggests that the first and second modal frequencies estimated by the transfer functions and the state space model are quite close to the ones obtained from the analytical method as $1.25 \mathrm{~Hz}$ and $2.89 \mathrm{~Hz}$. The stabilization diagram and the transfer functions for deeper layers such as TF A46-A6, TF A46-A11, and TF A46-A16 drew attention to existence of some mode(s) in the frequency range of $4.5-6.5 \mathrm{~Hz}$ and $5-6 \mathrm{~Hz}$. However, due to the multiband-band limited nature of the applied earthquake motion and its low frequency content beyond $4.5 \mathrm{~Hz}$ the third and the forth analytical modes at $4.53 \mathrm{and} 6.18 \mathrm{~Hz}$ were not adequately excited to be identified with confidence.

One way to verify the validity of the identified complex mode shapes, is to extract their real-valued counterparts known as normal mode shapes and compare them against those calculated using the available closed-form solutions proposed for simplified soil models in literature [28-31]. Normal modes are defined for structures with no damping or small damping. The phase angle distribution along the modal vector is either zero or 180 degree. The computation of normal modes from the identified complex modes for lightly damped structures or structures with proportional damping is a straight forward task as detailed in $[34,50]$. The challenge arises when the system is nonproportionally damped, and/or the set of identified modes is incomplete. Many studies have been published on nonproportionally damped systems where the number of DOFs and the identified modes were equal [33, 39]. Incomplete sets of identified modes are commonly encountered in the analysis of continuous structures. In principal, all structures are considered as spatially distributed systems with an infinite number of DOFs. When a distributed system is approximated by a lumped parameter system with countable number of DOFs, various incompleteness issues are often encountered as stated in [51]. Since it is not feasible to instrument all the DOFs, the identified mode shapes are only available at the measured DOFs. It is also possible that some modes are not excited or observed properly at the measuring points along the structure. Therefore, the number of identified modes could be larger or smaller than the number of sensors. The measurement locations certainly affect the quality and the spatial resolution of the identified mode shapes. The sensor location selection is an active research topic in modal testing, and wellstudied methods have been published in this area [52]. The optimal sensor selection is not within the scope of this paper; however, some attention will be paid to this subject. 
For cases with an insufficient number of sensors where some DOFs are left without instrumentation, a reduced second order model of the larger structure is created using the model reduction methods as explained in [53-55]. Another case of incompleteness arises where the number of modal coordinates, which is the same as the number of the identified modes, is smaller than the number of measured DOFs. A review on the existing methods treating this case can be found in [56-58]. Among them are methods that complete the set of the identified mode shapes by estimating the unidentified higher modes from an existing analytical or numerical model or by adding noise to the data to compensate for the rank deficiency problem [58]. All the proposed methods for extracting the normal modes from an incomplete set of identified complex modes are approximate to some extent. In this paper, a reduction transformation $[56,57]$ was implemented to estimate the normal modes from the three identified complex modes measured at five locations (A2, A6, A11, A16, A26). Herein, the number of sensors was higher than the number of identified modes. The reduction transformation solves the problem of modal truncation by reducing the number of eigenvector elements to the number of identified modes. The transformation matrix is obtained using the SVD technique which helps in finding and extracting the linear dependencies in the identified mode shapes. The theory of this method and the detailed procedure are described in [56, 57].

The identified modes were divided into 14 groups based on the similarities between their frequencies as shown in Fig. 4. Using a representative from each group, a total of 32 sets of complex mode shapes with 3 members were created where each member corresponds to one of the three modes identified around $1 \mathrm{~Hz}, 3 \mathrm{~Hz}$ and in the frequency range of 4.5-6.5 Hz. Using the reduction transformation the associated normal mode shapes were estimated, and it was found that only the normal mode shapes of the first two identified modes associated with lower frequencies could be estimated with reasonable accuracy as plotted in Fig. 8. In another attempt, two-member sets with complex modes belonging to the first and second modes around $1 \mathrm{~Hz}$ and $3 \mathrm{~Hz}$ were considered and the third mode in the frequency range $4.5-6.5 \mathrm{~Hz}$ was ignored. No two-member set was able to yield a reliable estimate of the second mode shape which implies that although the third identified mode was weakly excited it has a significant impact on the identification of the second mode. Of these 32 sets, one set was qualified upon evaluating the response prediction of the soil system as will be discussed in details in the next section. In this set the first and the second modes were selected from the diagrams for the model order 8 while the third mode was selected from the diagrams for the model order 12 where 2000 samples were used in the identification. The selected modal parameters are listed in Table 1.

Table 1. Identified Modal Parameters from the First Event

\begin{tabular}{|c|c|c|c|c|c|c|}
\hline Modes & \multicolumn{2}{|c|}{ First mode } & \multicolumn{2}{c|}{ Second mode } & \multicolumn{2}{c|}{ Third mode } \\
\hline $\begin{array}{c}\text { Undamped } \\
\text { frequencies }\end{array}$ & \multicolumn{2}{|c|}{1.35} & \multicolumn{2}{c|}{2.87} & \multicolumn{2}{c|}{6.20} \\
\hline Damping ratio & \multicolumn{2}{|c|}{0.13} & \multicolumn{2}{c|}{0.36} & \multicolumn{2}{c|}{0.37} \\
\hline \multirow{2}{*}{ Sensors } & $\begin{array}{c}\text { Complex mode } \\
\text { shape }\end{array}$ & \multicolumn{2}{c|}{$\begin{array}{c}\text { Complex mode } \\
\text { shape }\end{array}$} & \multicolumn{2}{c|}{$\begin{array}{c}\text { Complex mode } \\
\text { shape }\end{array}$} \\
\cline { 2 - 8 } & Amp. & phase & Amp. & phase & Amp. & phase \\
\hline A26 & 1 & 0 & 0.67 & 155.30 & 0.32 & 29.88 \\
\hline A16 & 0.33 & 19.20 & 1 & 0 & 1 & 0 \\
\hline A11 & 0.09 & 72.29 & 0.40 & 48.11 & 0.37 & 124.38 \\
\hline A6 & 0.06 & 87.65 & 0.25 & 61.31 & 0.22 & 152.90 \\
\hline A2 & 0.04 & 93.00 & 0.10 & 72.63 & 0.08 & 172.11 \\
\hline
\end{tabular}

As can be seen from Table 1, the first, second and the third identified modes are compatible with the first, second and fourth analytical modes at frequencies $1.25,2.89$, and $6.18 \mathrm{~Hz}$ while the third analytical mode at $4.53 \mathrm{~Hz}$ was missed out by both identification methods. By looking at the frequency content of the input motion presented in Fig. 2 (b) for the first event, it can be seen that the Fourier amplitude of the input signal is very low for the frequencies around $4.5 \mathrm{~Hz}$. Therefore, the analytical mode at $4.53 \mathrm{~Hz}$ was not adequately excited to be identified by the identification algorithm. 
The complex displacement mode shapes at the sensor locations are neither in phase nor 180-degrees out of phase. The associated normal modes extracted by implementing the reduced transformation are marked in Fig. 8 by broken lines. The mode shape amplitudes at soil surface was obtained by extrapolation. In comparing the identified normal modes with the analytical mode shapes calculated using equation 2.148 in reference [29] it appears that the first and second modes of our layered soil resemble the first and the second modes of the inhomogeneous soil model as shown in Fig. 7(b). However, the peak displacement for the second identified mode shape occurs in deeper layers compared with its analytical counterpart. Sparse instrumentation in the upper soil layers specifically between A16 and A26 forced the peak of the second identified mode shape to occur at the location of A16. Insufficient number of sensors in the upper soil layers where the third and higher analytical modes show a great deal of variation in the amplitude and sign can explain the reason why the third analytical mode was missed out by the 4SID algorithm. This emphasizes the impact of spatial distribution of sensors on the quality of the identified mode shapes. Another difference between the analytical mode shapes and their identified counterparts was observed as small fluctuations in the identified mode shapes close to the interface of the clay and sand layers. This is likely due to the strong stiffness contrast between the soft clay layer and the underlying dense sand layer as reflected in the shear wave velocity profile shown in Fig. 7(a). Also it should be noted that in obtaining the analytical mode shapes identical damping ratios was assumed for all modes (1\%) while the damping ratio of the first and second identified modes was estimated as $13 \%$ and $36 \%$, respectively. The analytical mode shapes were found to be less sensitive to the variations in the parameters of the exponential model of the shear wave velocity profile as opposed to the analytical natural frequencies. Changing $V_{0}$ from 12 to $20 \mathrm{~m} / \mathrm{s}$ did not cause a significant change in the location of the maximum displacements. Validation of the identified parameters presented in this section highlights the benefit in considering a combination of analytically and experimentally identified models for validating the results and thereby avoiding the missing modes.

\section{Response prediction}

In synthesizing the state space prediction model described in Eqs. (5) and (6) from the identified modes, we confronted two obstacles. First, the set of the identified complex mode shapes as mentioned in the previous section was incomplete and as a result the scaling of the complex mode shapes in Eqs. (3) and (4) could not be performed. Second, the input influence matrix $\boldsymbol{\beta}$ containing the lumped masses at the measured DOFs was unknown. The first issue was overcome by taking only three elements of the complex eigenvectors (Table 1) into account as if the soil response was recorded by only three sensors. Accordingly, eight different sensor combinations named as Trials 1-8 listed in Table 2 were selected. As shown by De Angelis and Imbimbo [39], a complete set of mode shapes can be scaled provided that the mass of only one DOF is known. A priori knowledge of the mass matrix, even partially, was also realized by Kim and Lynch [10] for conversion of an identified black-box state space model to its physically meaningful gray-box model. In this regard, to treat the second issue, the lumped mass at only one sensor location was approximated based on the unit weight and the thickness of the corresponding soil layer. Following the procedure detailed in [39], the resultant mass matrices estimated from the scaled mode shapes as stated in Eq. (17) for all trials appeared with one or two negative diagonal entries. This implies that the selected set of measurements in the trials could not be generated by a 3DOFs lumped mass system. In other words, synthesizing the state space prediction model using the incomplete set of modal parameters results in a model which may not correspond to a physical system. This depends on the contribution of the missed out modes in the system response. Yet, using the calculated mass matrix containing the negative entries and the scaled complex mode shapes, the state space model of each trial was synthesized and utilized to predict the soil response at the selected sensor locations as depicted in Fig. 9.

Those models that reproduced the peak accelerations within 5-10\% error and the lowest root mean square of the residual between the measured and predicted values were selected as successful trials. As can be seen in Fig. 9, time histories at A2 in Trial 3, A6 and A26 in Trial 5, and A11 and A16 in Trial 6 were closely approximated by the corresponding models. It is noteworthy to mention that another set of analyses were performed considering only the first two identified modes and the response at all sensor locations were predicted using the two-sensor combinations. The results are not reported in this paper due to space limitation, but overall no remarkable difference was observed between the two cases; however surprisingly no two-sensor combination could result in an acceptable prediction at A2 in the first sand layer. It can be inferred that the third identified mode played a major role in the response of this sensor. The contributions of individual modes to the overall response at each location are illustrated in Fig. 10. In 
spite of the fact that the first mode did not contribute appreciably to the responses at A2, A6, A11 and A16, it predicted the total response at A26 very well. Examination of the transfer functions in Fig. 6 (a) confirms that first mode is more pronounced in the response of the soil in the upper most layer while in the deeper layers the second and the third modes were more influential.

The most basic use of an identified system is to simulate the system response to various inputs. To validate our identified models in each trial shown in Table 2, the response of the soil system to the second and the third shaking events shown in Fig. 2 were simulated using the predictive models. The simulated time histories and the corresponding Fourier amplitude spectra are compared against the measured responses in Figs. 11 and 12 for the second and the third events, respectively. The results indicate that the identified models from the first event can predict the soil response at A2, A6 in the dense sand layers, and A11 in the first clay layer very well in the second event while the prediction accuracy is lower for shallower sensors A16 and A26 in the second and fourth clay layers, respectively. For the third event, the models were able to capture the maximum acceleration along with the frequencies carrying the major part of the energy at A2, A6 and A11 very well, the response at A16 and A26 were, however, highly over estimated.

Overall, the prediction capabilities of the models can be considered reasonable except in the upper clay layers and under strong motion such as the third shaking event. It should be pointed out here that the range of application of the identified models is limited to linear systems, while the soil deposit, particularly in the upper layers under strong excitation, is expected to behave in a nonlinear and time-varying manner. Moreover, relatively higher frequency content of the third event beyond $4 \mathrm{~Hz}$ and the absence of the higher modes in this frequency range except the third identified mode at frequency $6.2 \mathrm{~Hz}$ is another reason for the poor prediction of response in the Kobe shaking event.

Table 2. Sensor Configuration

\begin{tabular}{|c|c|c|}
\hline Trials & Sensor combinations & $\begin{array}{c}\text { Satisfactory } \\
\text { predictions }\end{array}$ \\
\hline Trial 1 & Triple (A2, A6, A26) & - \\
\hline Trial 2 & Triple (A2, A11, A26) & - \\
\hline Trial 3 & Triple (A2, A16, A26) & A2 \\
\hline Trial 4 & Triple (A6, A11, A26) & - \\
\hline Trial 5 & Triple (A6, A16, A26) & A6, A26 \\
\hline Trial 6 & Triple (A11, A16, A26) & A11, A16 \\
\hline Trial 7 & Triple (A2, A6, A16) & - \\
\hline Trial 8 & Triple (A2, A11, A16) & - \\
\hline
\end{tabular}

\section{Conclusions}

In this paper, we applied the subspace state space system identification (4SID) method, for the first time, to estimate the modal quantities of a layered soil deposit subjected to a base motion. Three modes were identified using the data acquired in the first small shaking event from five accelerometers distributed within the soil deposit. The identified modal frequencies were compared to the peaks of transfer function spectra, and good comparisons were obtained. The normal mode shapes were extracted from the identified complex counterparts and were compared to those obtained from a published analytical model in the frequency range of interest. In spite of the difficulty in obtaining the necessary parameters for the analytical model, the extracted normal mode shapes corresponding to the first and second identified modes matched their counterparts calculated from the analytical model in a qualitative sense. A denser array of accelerometers particularly in the middle and in the surficial layers would have resulted in better estimate of modal quantities since the sparse instrumentation in this region shifted the peak in the second 
identified mode shapes to the deeper layers. Comparison of the identified and theoretical amplification functions revealed the weakly excited modes in the identification process due to the insufficient instrumentation and narrowband frequency content of the excitation motion.

The assembled state space models using the incomplete set of identified modes were utilized to predict the responses during three shaking events. These models predicted the acceleration-time histories and the corresponding Fourier response spectra reasonably well for the small first and moderate second shaking events. Although the proposed models' prediction accuracy was lower in the upper layers for the strong third shaking event, it was still able to predict the behavior of the deeper layers reasonably well. Relatively higher frequency content of the third event beyond $4 \mathrm{~Hz}$ and the absence of the third analytical mode in the identified modal parameters set is one reason for the poor prediction of response in the third shaking event. It is also believed that the upper soft clay layers behaved in a nonlinear and time-varying manner during the third event. It is, however, interesting to note that a discrete model developed for a linear system was able to make reasonably accurate predictions for a continuous soil deposit even in a moderately nonlinear range.

The results highlight the benefit in considering a combination of an analytical model and an experimentally identified model for validating the results and thereby avoiding missing modes. Accordingly, it is recommended several system identification methods using input excitations with different frequency contents be considered in appreciation of the merits and limitations of each system identification approach.

\section{Acknowledgements}

This project was funded by a U.S, National Science Foundation (NSF) George E. Brown, Jr. Network for Earthquake Engineering Simulation Research (NEESR) grant (Grant No. CMMI-0830328) and this support is gratefully acknowledged. Any opinions, findings, and conclusions or recommendations expressed in this paper are those of the authors and do not necessarily reflect the views of NSF. We would also like to acknowledge valuable suggestions provided by two anonymous reviewers that helped us improve this paper.

\section{References}

[1] Lus, H., Betti, R. and Longman R. W., "Identification of linear structural systems using earthquake-induced vibration data," Earthquake Engineering and Structural Dynamics, 1999, Vol. 28:1449-1467.

[2] Arici, Y. and Mosalam, K.M., "Modal identification of bridge systems using state-space methods," Structural Control and Health Monitoring, 2005, Vol. 12: 381-404.

[3] Siringoringo, D. M. and Fujino Y., "Dynamic characteristics of a curved cable-stayed bridge identified from strong motion records," Engineering Structures, 2007, Vol. 29: 2001-2017.

[4] Siringoringo, D. M. and Fujino Y., "System identification applied to long-span cable-supported bridges using seismic records, Earthquake Engineering and Structural Dynamics," 2008, Vol. 37: 361-386.

[5] Fraraccio, G., Brügger. A., and Betti, R., "Identification and damage detection in structures subjected to base excitation," Experimental Mechanics, 2008, Vol. 48: 521-528.

[6] He. X., Moaveni, B., Conte, J. P., Elgamal, A. and Masri, S. F., "System identification of Alfred Zampa Memorial Bridge using dynamic field test data," Journal of Structural Engineering, 2009, Vol. 135 (1): 54-66.

[7] Peeters, B. and Ventura, C. E., "Comparative study of modal analysis techniques for bridge dynamic characteristics," Mechanical Systems and Signal Processing, 2003, Vol.17 (5), pp: 965-988.

[8] Reynders, E. and De Roeck, G., "Referenced-based combined deterministic-stochastic subspace identification for experimental and operational modal analysis," Mechanical Systems and Signal Processing, 2008, Vol. 22: 617-637.

[9] Weng, J., Loh, C., Lynch, J. P., Lu, K., Lin, P., and Wang, Y., "Output-only modal identification of a cable stayed bridge using wireless monitoring systems," Engineering Structures, 2008, Vol. 30: 1820-1830.

[10] Kim, J. and Lynch J. P., "Subspace system identification of support-exited structures-Part II: gray-box interpretations and damage detection," Earthquake Engineering and Structural Dynamics, 2012, Vol. 41, Issue 15: 2253-2271.

[11] Gazetas, G., "Vibrational characteristics of soil deposits with variable wave velocity," International Journal for Numerical and Analytical Methods in Geomechanics, 1982, Vol. 6:1-20. 
[12] Kramer, S.L., "Geotechnical Earthquake Engineering," Prentice Hall, N.J., 1996.

[13] Hadjian, A. H., "Fundamental period and mode shape of layered soil profiles," Soil Dynamic and Earthquake Engineering, 2002, 22:885-891.

[14] Dezi, F., Carbonari, S., and Leoni, G., "Static equivalent method for the kinematic interaction analysis of single piles," Soil Dynamics and Earthquake Engineering, 2010, 30: 679-690.

[15] Zeghal, M. and Elghamal, A. W., "Site response and vertical seismic arrays," Progress in Structural Engineering and Materials, 2000, Vol. 2(1): 92-101.

[16] Brennan, A. J., Thusyanthan, N. I. and Madabhushi, S. P. G., "Evaluation of shear Modulus and damping in dynamic centrifuge tests," Journal of Geotechnical and Geoenvironmental Engineering, 2005, Vol. 131(12):1488-1497.

[17] Conti, R., and Viggiani, G. M. B., "Evaluation of soil dynamic properties in centrifuge tests," Journal of Geotechnical and Geoenvironmental Engineering, 2012, Vol. 138(7):850-859.

[18] Afacan, K. B., Brandenberg, S. J., and Stewart, J. P., "Centrifuge modeling studies of site response in soft clay over wide strain range," Journal of Geotechnical and Geoenvironmental Engineering, 2014, Vol. 140(2):1-13

[19] Dobry, R. and Whitman, R. V., "Effect of Local Soil Conditions Upon Earthquake Damage," Progress Report, School of Engineering, Department of Civil Engineering, Massachusetts Institute of Technology, 1969.

[20] Elgamal, A. W., Zeghal, M., Tang, H. T., and Stepp, J. C., "Lotung down-hole array, I. Evaluation of site dynamic properties," Journal of Geotechnical Engineering, 1995, Vol. 121 (4): 350-362.

[21] Elgamal, A. W., Zeghal, M., Parra, E., Gunturi, R., Tang, H. T., and Stepp, J. C., "Identification and modeling of earthquake ground response, I. Site amplification," Soil Dynamics and Earthquake Engineering,1996, Vol. (15): 499-522.

[22] Zeghal, M., Elgamal, A.-W., Tang, H. T. and Stepp, J.C., "Lotung downhole array. II: Evaluation of soil nonlinear properties," Journal of Geotechnical Engineering, 1995, Vol. 121 (4):363-378.

[23] Glaser, S.D., "System identification and its application to estimating soil properties," Journal of Geotechnical Engineering, 1995, Vol. 121 (7): 553-560.

[24] Glaser, S. D., "Insight into liquefaction by system identification," Geotechnique, 1996, Vol. 46 (4): 641-655.

[25] Glaser, S. D. and Baise, L. G., "System identification estimation of soil properties at Lotung site," Soil Dynamics and Earthquake Engineering, 2000, Vol. 19: 521-531.

[26] Kwok, A., Stewart J. P., Hashash, Y., Matasovic, N., Pyke, R., Wang, Z., and Yang, Z., "Use of exact solutions of wave propagation problems to guide implementation of nonlinear seismic ground response analysis procedures," Journal of Geotechnical and Geoenvironmental Engineering, 2007, Vol. 133 (11):1385-1398.

[27] Hashash, Y. and Park, D., "Viscous damping formulation and high frequency motion propagation in non-linear site response analysis," Soil Dynamics and Earthquake Engineering, 2002, Vol. 22:611-624.

[28] Dobry, R., Oweis, I. and Urzua, A., "Simplified procedures for estimating the fundamental period of a soil profile," Bulletin of Seismological Society of America, 1976, Vol. 66 (4):1293-1321.

[29] Schulze K., "Vibrational characteristics and seismic response of inhomogeneous soils," MSc thesis, 2005, Department of Civil Engineering, City University of New York.

[30] Rovithis, E. N., Parashakis, H. and Mylonakis, G. E.,"1D harmonic response of layered inhomogeneous soil: Analytical investigation," Soil Dynamic and Earthquake Engineering, 2011, Vol. 31:879-890.

[31] Vrettos, C., "Dynamic response of soil deposits to vertical SH waves for different rigidity depth-gradients," Soil Dynamics and Earthquake Engineering, 2013, Vol. 47: 41-50.

[32] Alvin, K. F., Robertson, A. N., Reich, G. W., and Park, K. C., "Structural system identification: from reality to models," Computers and Structures, 2003, Vol. 81: 1149-1176.

[33] Balmes, E., "New results on the identification of normal modes from experimental complex modes," Mechanical Systems and Signal Processing, 1997, Vol. 11 (2): 229-243.

[34] Alvin, K. F., Peterson, L. D., and Park, K. C., "Extraction of normal modes and full modal damping from complex modal parameters," American Institute of Aeronautics and Astronautics Journal, 1997, Vol. 35 (7):1187-1194

[35] Viberg, M., "Subspaced-based methods for the identification of linear time-invariant systems," Automatica, 1995, Vol. 31 (12): 1835-1851.

[36] Van Overschee, P. and De Moor, B., "Subspace Identification for Linear Systems: Theory-ImplementationApplication," 1996, Dordrecht, The Netherlands: Kluwer Academic Publishers.

[37] Kim, J. and Lynch J. P., "Subspace system identification of support-exited structures-Part I: theory and blackbox system identification," Earthquake Engineering and Structural Dynamics, 2012, Vol. 41, Issue 15: 22352251.

[38] Juang, J-N, "Applied System Identification," Prentice Hall, 1993. 
[39] De Angelis, M. and Imbimbo, M., "A procedure to identify the modal and physical parameters of a classically damped system under seismic motions," Advances in Acoustics and Vibration, 2012, Article ID 975125.

[40] De Angelis, M., Lus, H., Betti, R., and Longman, R. W., "Extracting physical parameters of mechanical models from the identified state-space representations," Journal of Applied Mechanics, 2002, Vol. 69:617-625.

[41] Liu, C., Soltani, H., Pinilla, J. D., Muraleetharan, K.K., Cerato, A.B., and Miller, G.A., "Centrifuge investigation of seismic behavior of pile foundations in soft clays," GSP No. 211, Proceedings (CDROM), Geo-Frontiers 2011: Advances in Geotechnical Engineering, Geo-Institute, ASCE, Dallas, Texas, March, 585594.

[42] Reynders, E., "System identification methods for (operational) modal analysis: review and comparison," Archives of Computational Methods in Engineering, 2012, Vol. 19, Issue 1: 51-124.

[43] Maciejowski, J. M., "Guaranteed stability with subspace methods" Systems and Control Letters, 1995, Paper \#2694.

[44] Chui, N. L. C. and Maciejowski, J. M., "Realization of stable models with subspace methods," Automatica, 1996, Vol. 32(11): 1587-1595.

[45] Gestel, T. V., Suykens, J. A. K., Van Dooren, P., and De Moor, B., "Identification of stable models in subspace identification by using regularization," IEEE Transactions on Automatic Control, 2001, Vol. 46(9): 1416-1420.

[46] Lacy, S. L. and Bernstein, D. S., "Subspace identification with guaranteed stability using constrained optimization," IEEE Transactions on Automatic Control, 2003, Vol. 48(7): 1259-1263.

[47] Stoica, P. and Moses, R. L., "Spectral Analysis of Signals," Upper Saddle River, N.J. : Pearson Prentice Hall, 2005. Chapters 1 and 2.

[48] Safak, E., "Models and methods to characterize site amplification from a pair of records," Earthquake Spectra, 1997, Vol. 13 (1): 97-129.

[49] Mikami, A., Stewart, J. P., and Kamiyama, M., "Effects of time series analysis protocols on transfer functions calculated from earthquake accelerograms," Soil Dynamic and Earthquake Engineering, 2008, Vol. 28, :695706.

[50] Alvin, K. F., and Park, K. C., "Second-order structural identification procedure via state-space-based system identification" AIAA Journal, 1994, Vol. (32), No. 2: 397-406.

[51] Mukhopadhyay, S., Lus, H., and Betti, R., "Modal parameter based structural identification using input-output data: Minimal instrumentation and global identifiability issues," Mechanical System and Signal Processing, 2014, Vol. 45:283-301.

[52] Udwadia, F., "Methodology for optimum sensor locations for parameter identification in dynamic system," Journal of Engineering Mechanics, 1994, Vol. 20 (2): 368-390.

[53] Alvin, K. F., Peterson, L.D., and Park, K. C., "Methods for determining minimum-order mass and stiffness matrices from modal test data," American Institute of Aeronautics and Astronautics Journal, 1995, Vol. 33 (1): 128-135.

[54] Lus, H., De Angelis, M., and Betti, R., "A new approach for reduced order modeling of mechanical systems using vibration measurements," Journal of applied mechanics, ASME, 2003, Vol. 70 (5): 715-723.

[55] Lus, H., De Angelis, M., Betti, R., and Longman, R. W., "Constructing second-order models of mechanical systems from identified state space realizations. Part I: Theoretical discussions, " Journal of Engineering Mechanics, ASCE, 2003, Vol. 129 (5): 477-488.

[56] Ibrahim, S. R. and Fuellekrug U., "Investigation into exact normalization of incomplete complex modes by decomposing transformation," Proceeding of the $8^{\text {th }}$ International Modal Analysis Conference, Kissimmee, FL, USA, 1990, pp: 205-212.

[57] Füellekrug, U., "Computation of real normal modes from complex eigenvectors, " Mechanical System and Signal Processing, 2008, Vol. 22: 57-65.

[58] Ibrahim, S. R., "Computation of normal modes from identified complex modes," AIAA Journal, 1983, Vol. 21 (3):446-451. 
Fig. 1. Centrifuge model of free-field soil in prototype scale (dimensions are in meter)

Fig. 2. Characteristics of the base motion in the first, second and third events, (a) time histories, (b) Fourier spectra

Fig. 3. Singular values plot (number of row blocks=12)

Fig. 4. Stabilization diagrams of modal frequencies and damping ratios for the first event, (a) base motion-time history, (b) model order=8, (c) model order=10, (d) model order=12

Fig. 5. Stabilization diagrams of complex mode shapes (first number in the legend specifies the model order and second number specifies the data length), (a) model order $=8$, (b) model order $=10$, (c) model order $=12$

Fig. 6. (a) Smoothed transfer functions for the first event, (b) Amplification functions of the soil model using the analytical method

Fig. 7. (a) Estimated shear wave velocity profile (b) Identified normal mode shapes in the first event labeled with (I) and the natural modes of an inhomogeneous layer over a rigid rock using analytical solution in [29] labeled with (A)

Fig. 8. Extracted normal mode shapes from different sets of complex mode shapes

Fig. 9. Response predictions for the first event,

(a) Trial 1, (b) Trial 2, (c) Trial 3, (d) Trial 4, (e) Trial 5, (f) Trial 6, (g) Trial 7, (h) Trial 8

Fig. 10. The responses of individual modes in the first event (a) A2, (b) A6, (c) A11, (d) A16, (e) A26

Fig. 11. Response predictions for the second event (a) time histories (b) Fourier amplitude spectra

Fig. 12. Response prediction for the third event (a) time histories (b) Fourier amplitude spectra 


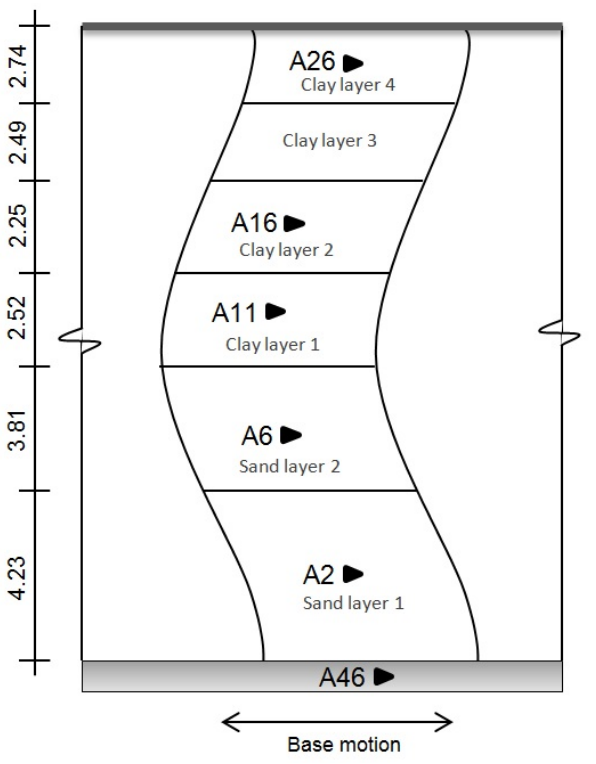

Fig. 1. Centrifuge model of free-field soil in prototype scale (dimensions are in meter)
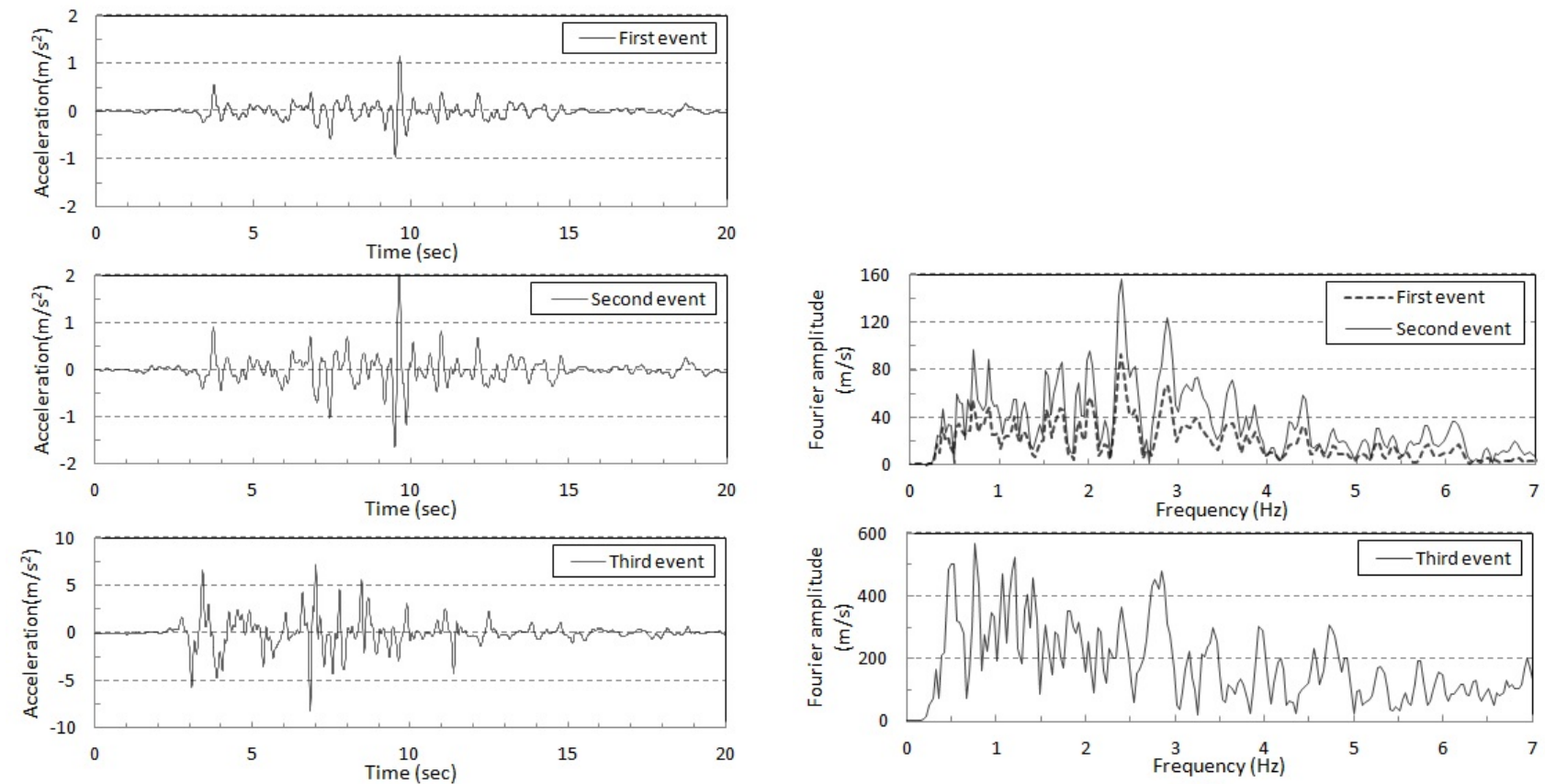

(a)

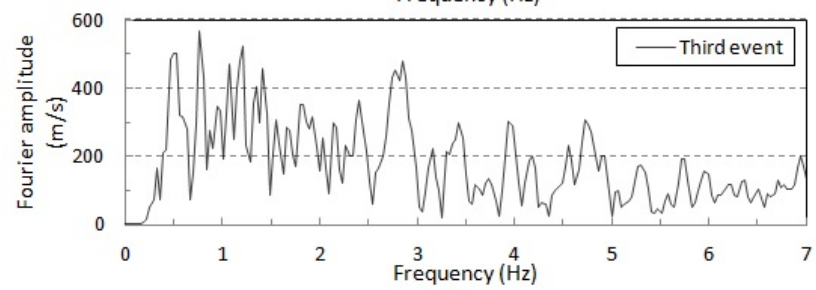

(b)

Fig. 2. Characteristics of the base motion in the first, second and third events, (a) time histories, (b) Fourier spectra

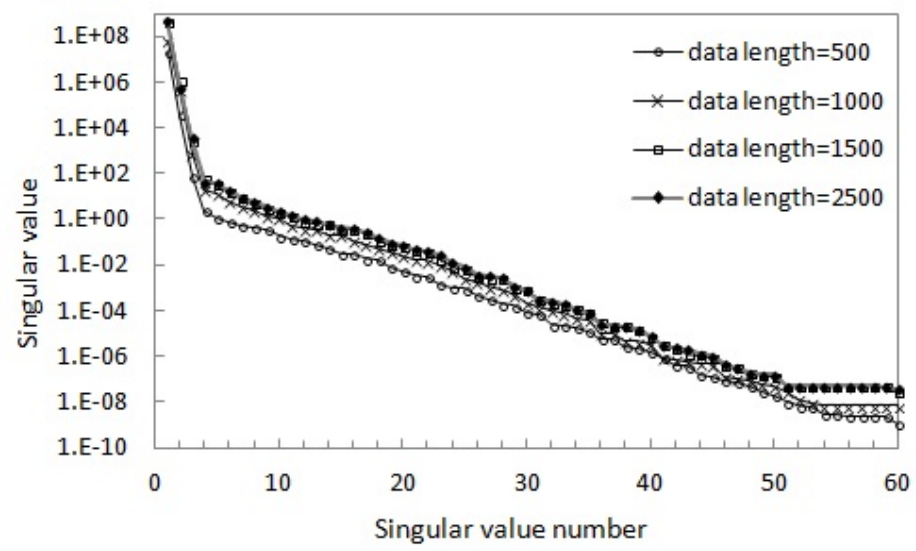

Fig. 3. Singular values plot (number of row blocks=12) 


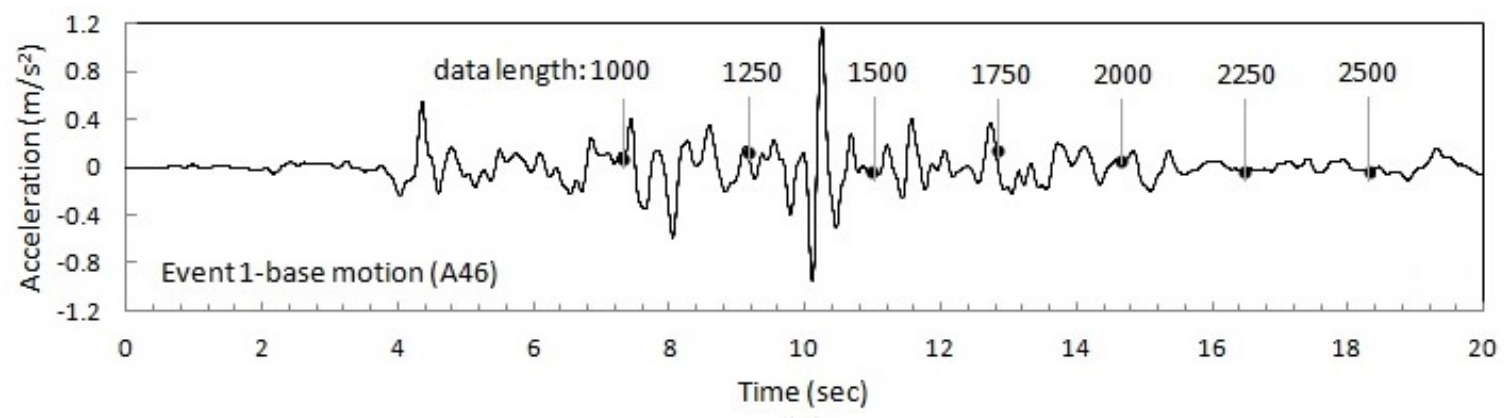

(a)
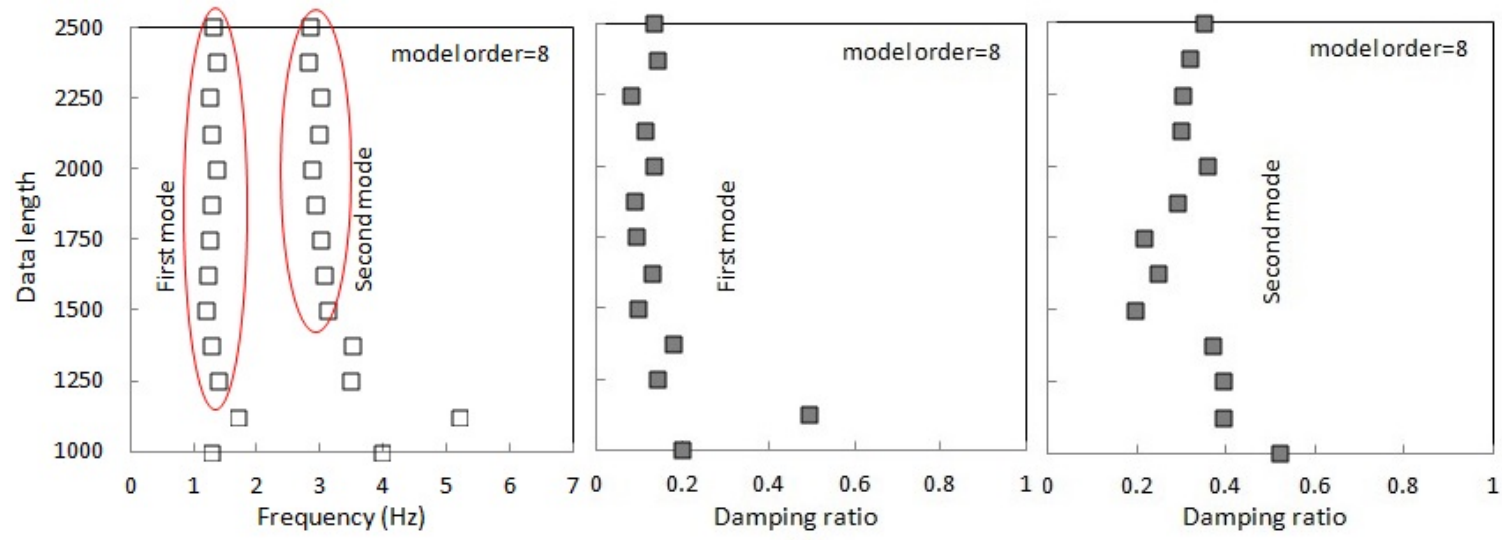

(b)
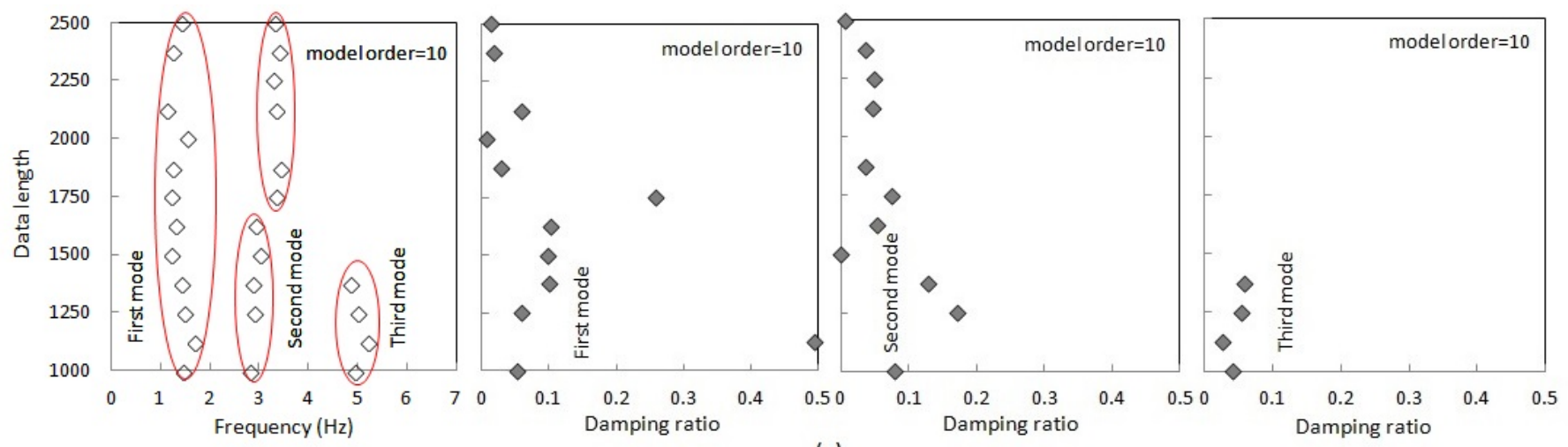

(c)
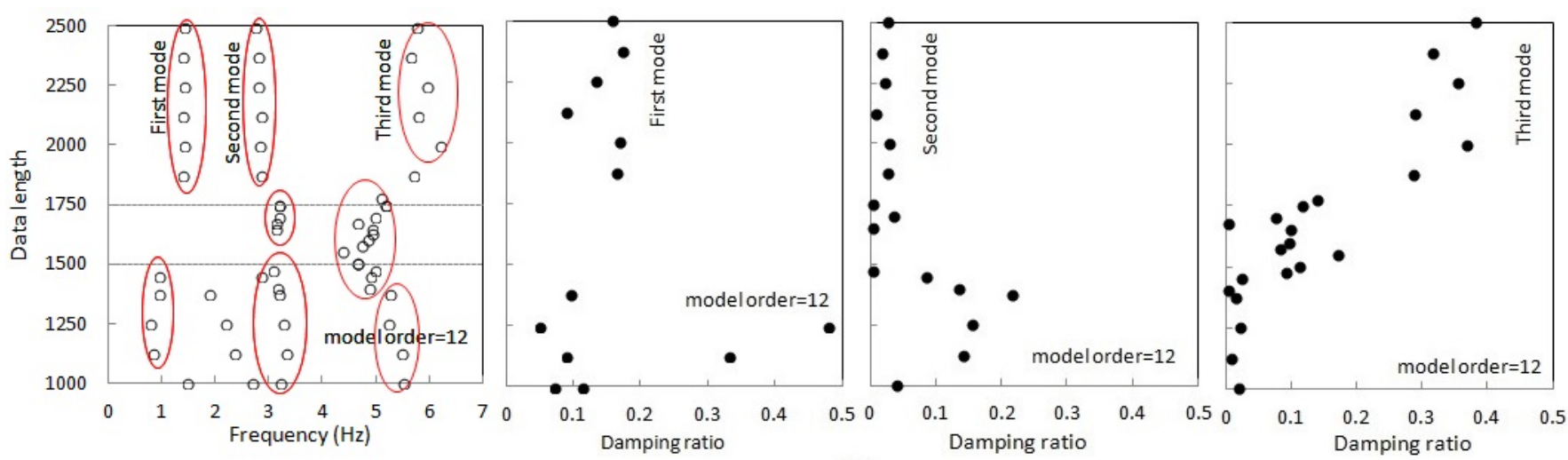

(d)

Fig. 4. Stabilization diagrams of modal frequencies and damping ratios for the first event, (a) base motion-time history, (b) model order $=8$, (c) model order $=10$, (d) model order $=12$ 

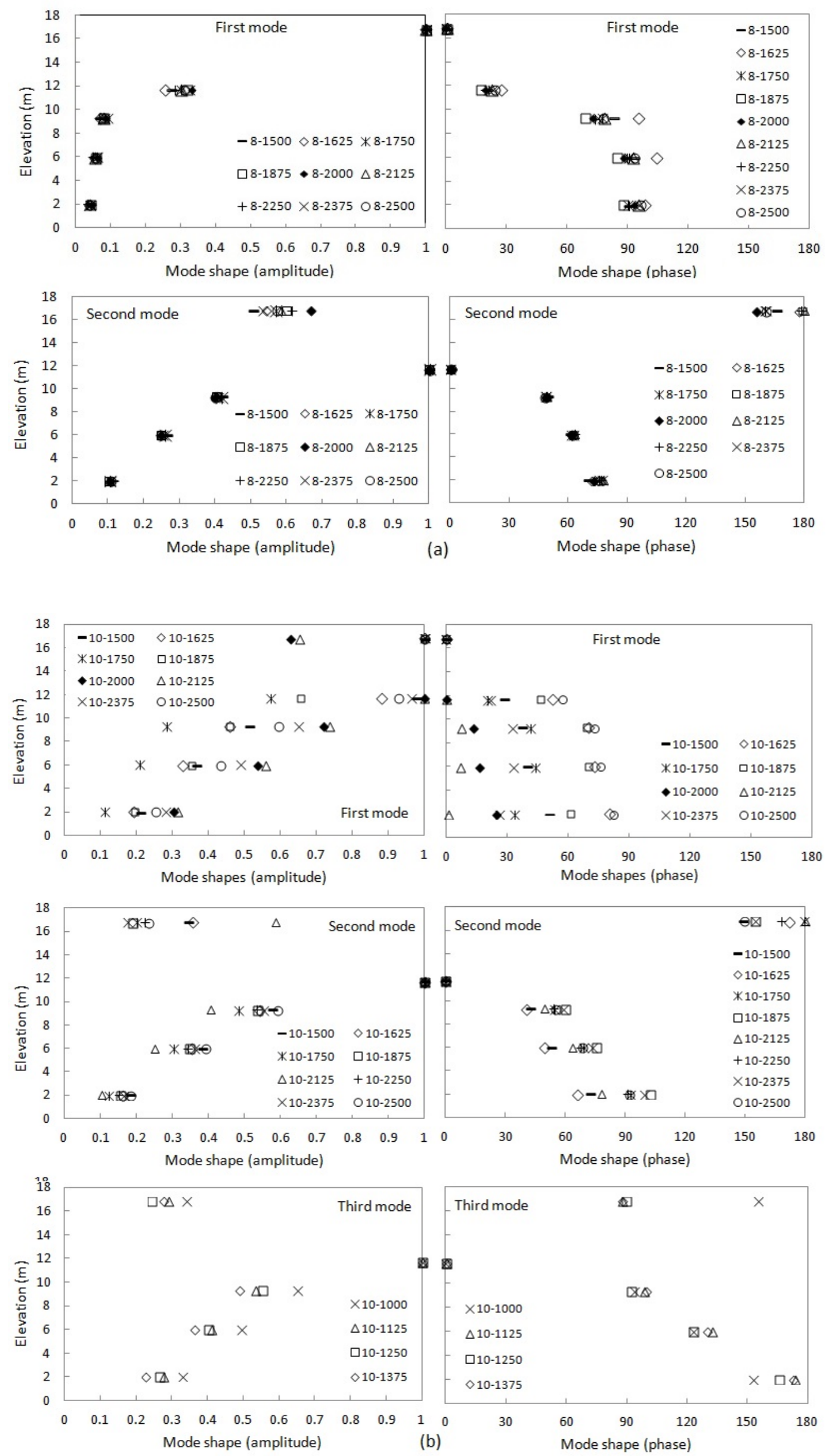

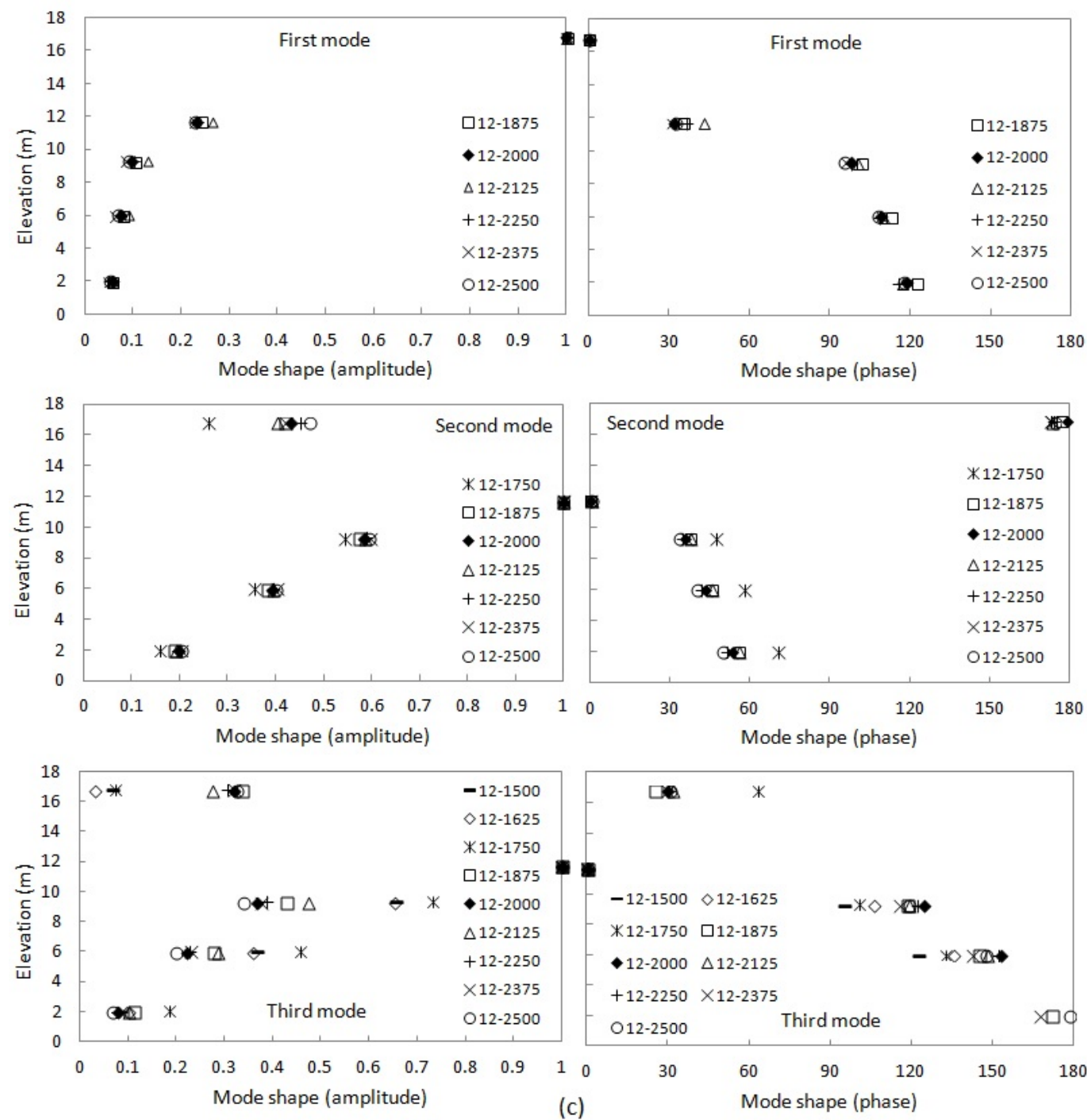

Fig. 5. Stabilization diagrams of complex mode shapes (first number in the legend specifies the model order and second number specifies the data length), (a) model order=8, (b) model order=10, (c) model order=12 


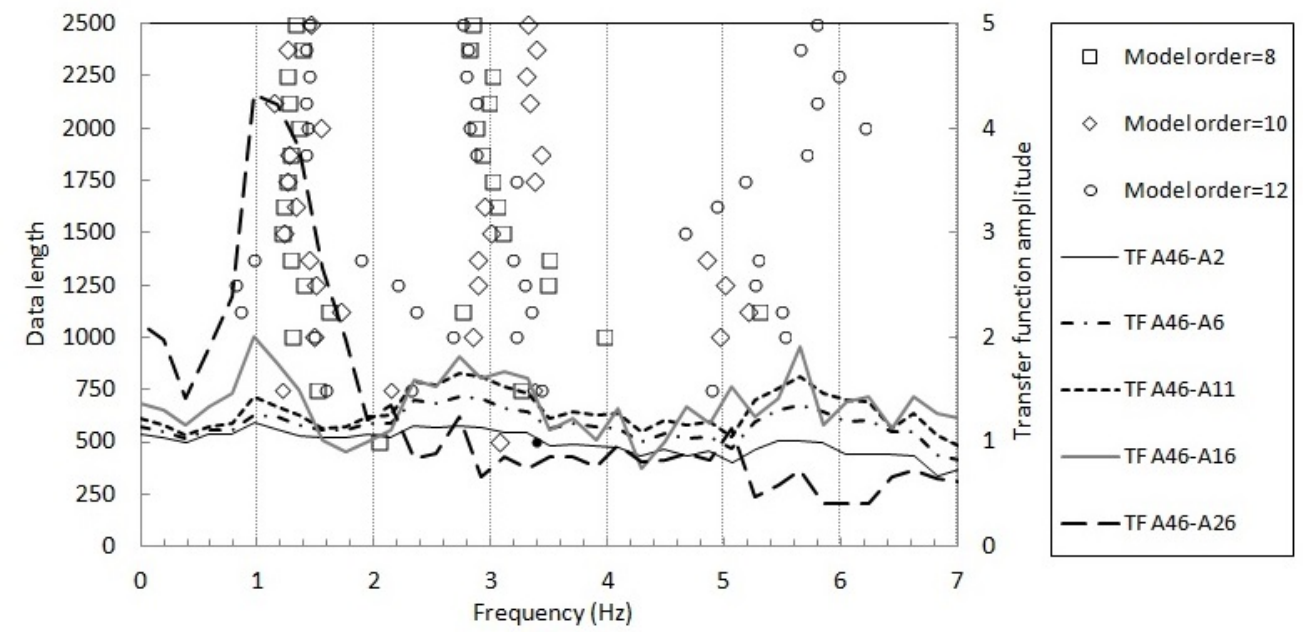

(a)

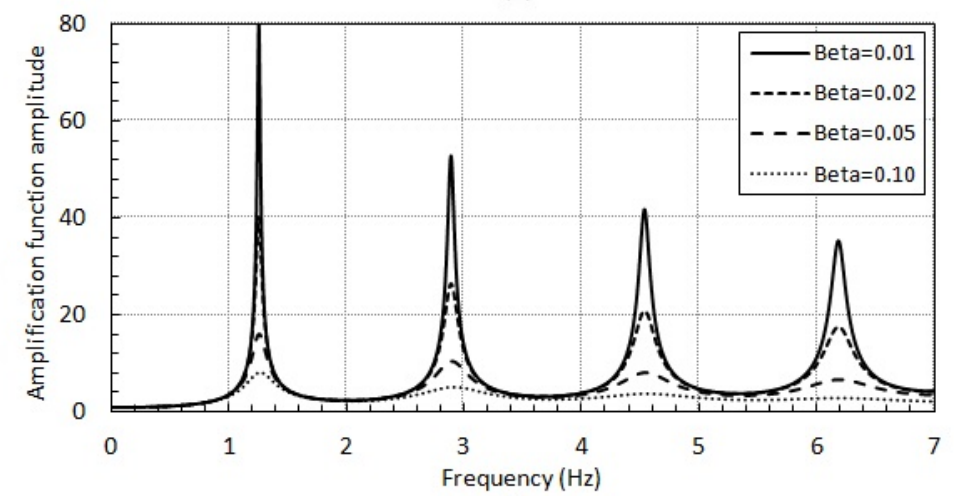

(b)

Fig. 6. (a) Smoothed transfer functions for the first event, (b) Amplification functions of the soil model using the analytical method

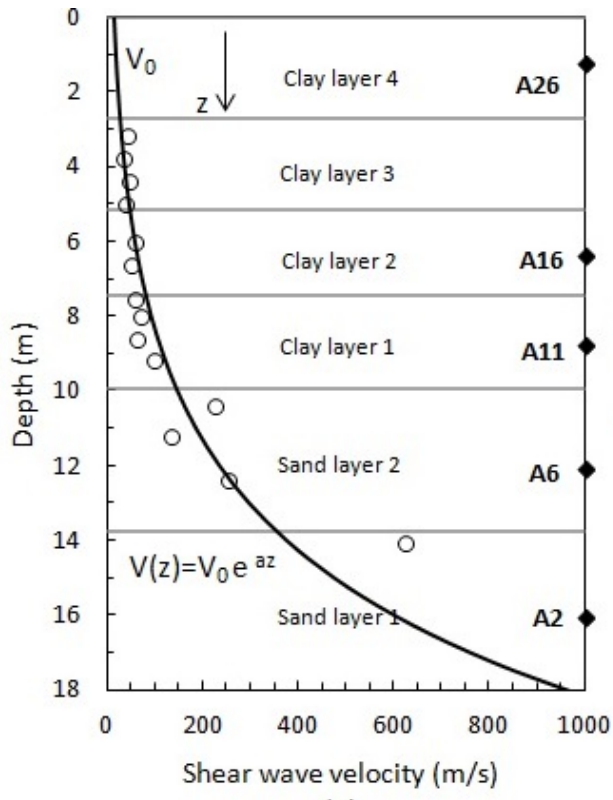

(a)

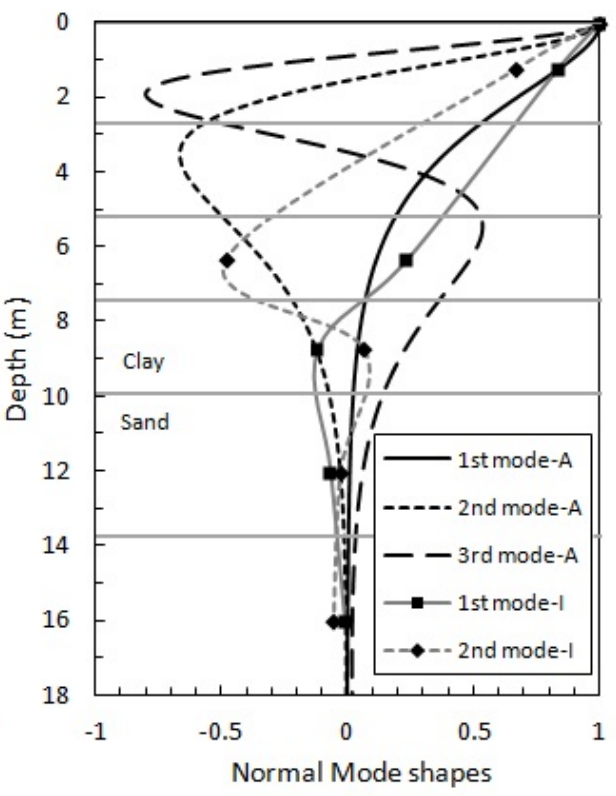

(b)

Fig. 7. (a) Estimated shear wave velocity profile (b) Identified normal mode shapes in the first event labeled with (I) and the natural modes of an inhomogeneous layer over a rigid rock using analytical solution in [29] labeled with (A) 


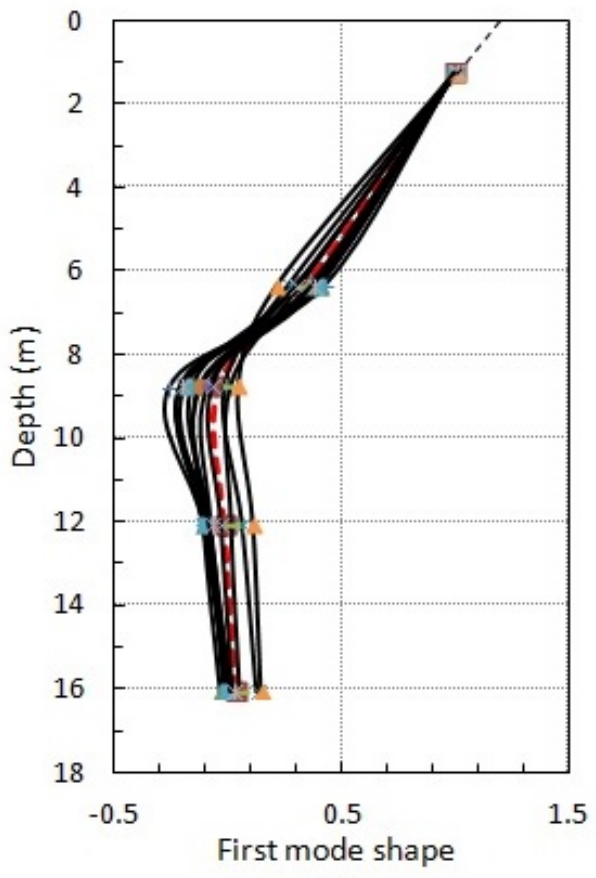

(a)

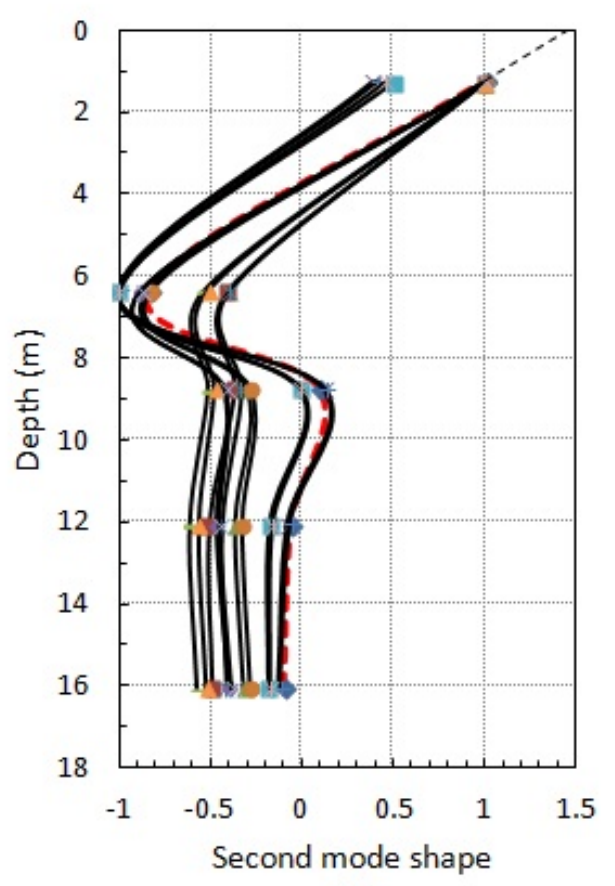

(b)

Fig. 8. Extracted normal mode shapes from different sets of complex mode shapes 

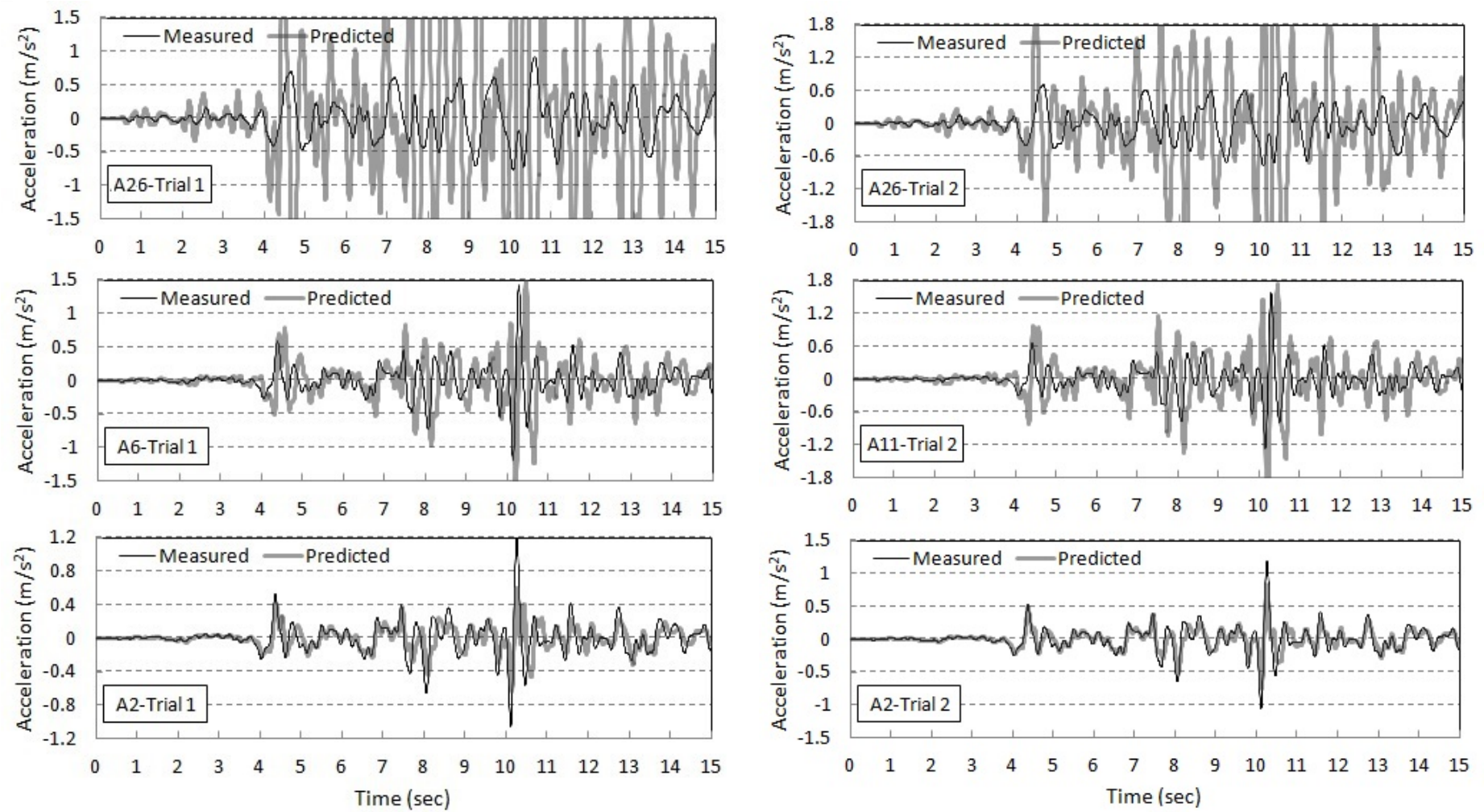

(a)

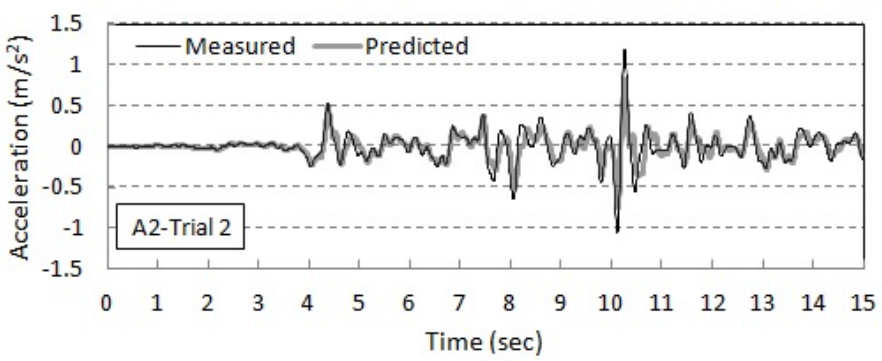

(b)
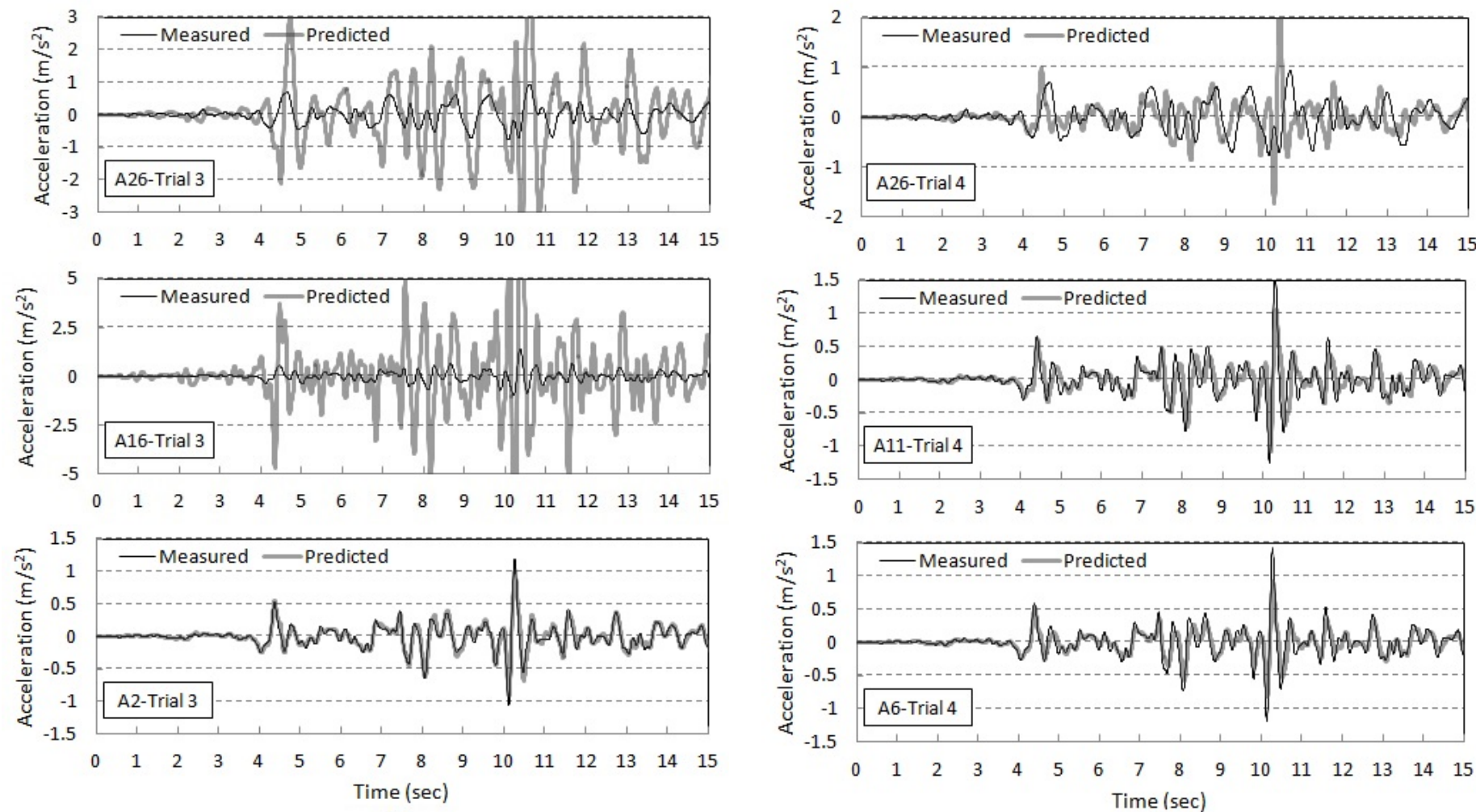

(c)

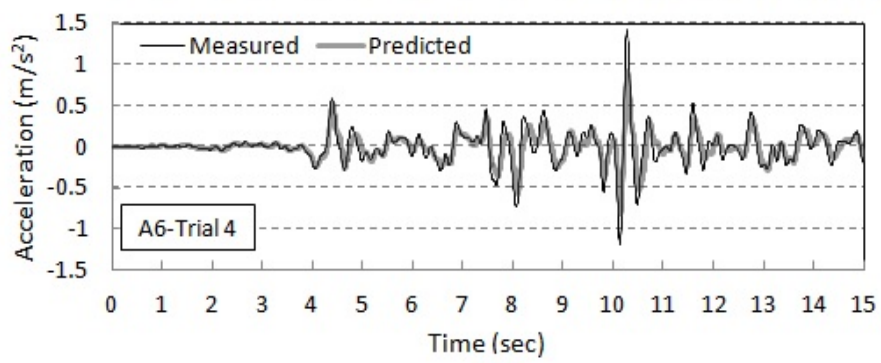

(d) 

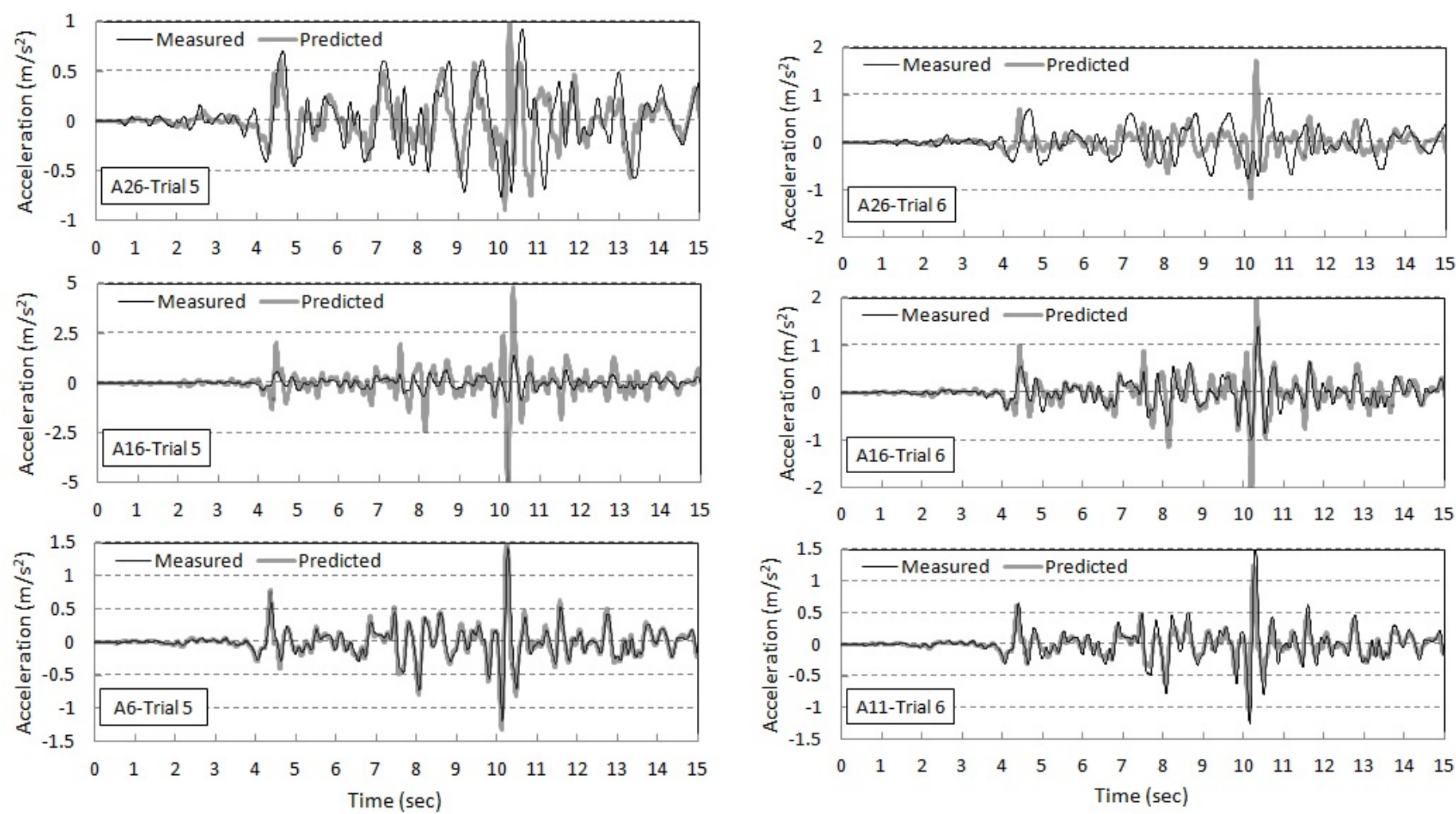

(e)

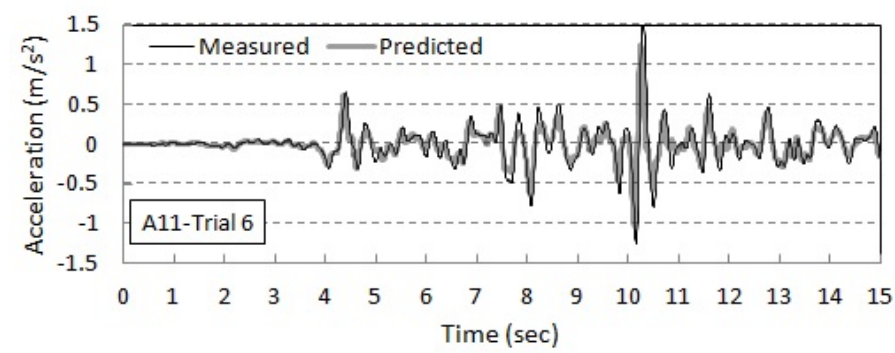

(f)
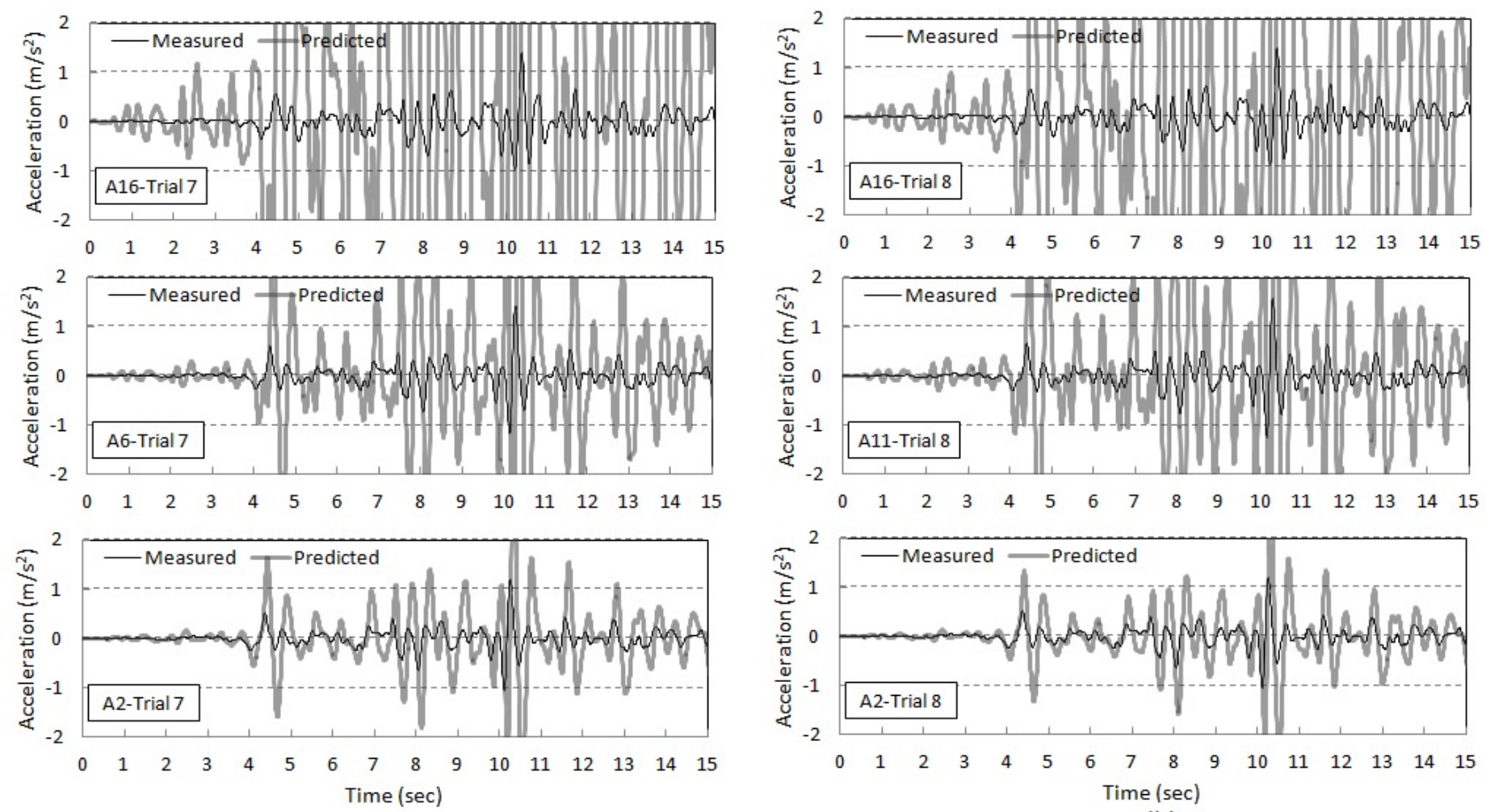

(g)

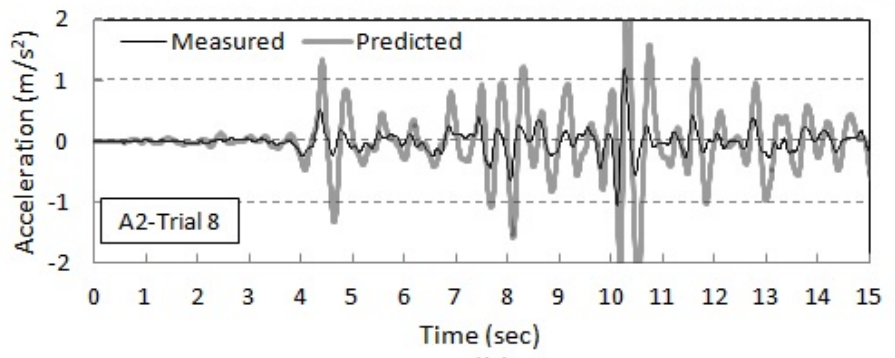

(h)

Fig. 9. Response predictions for the first event,

(a) Trial 1, (b) Trial 2, (c) Trial 3, (d) Trial 4, (e) Trial 5, (f) Trial 6, (g) Trial 7, (h) Trial 8 


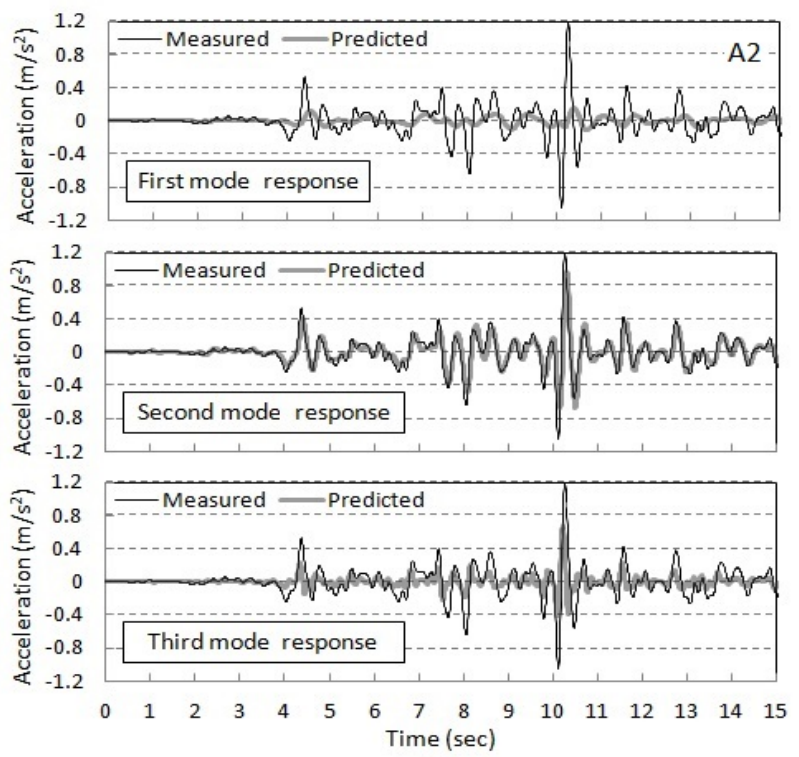

(a)

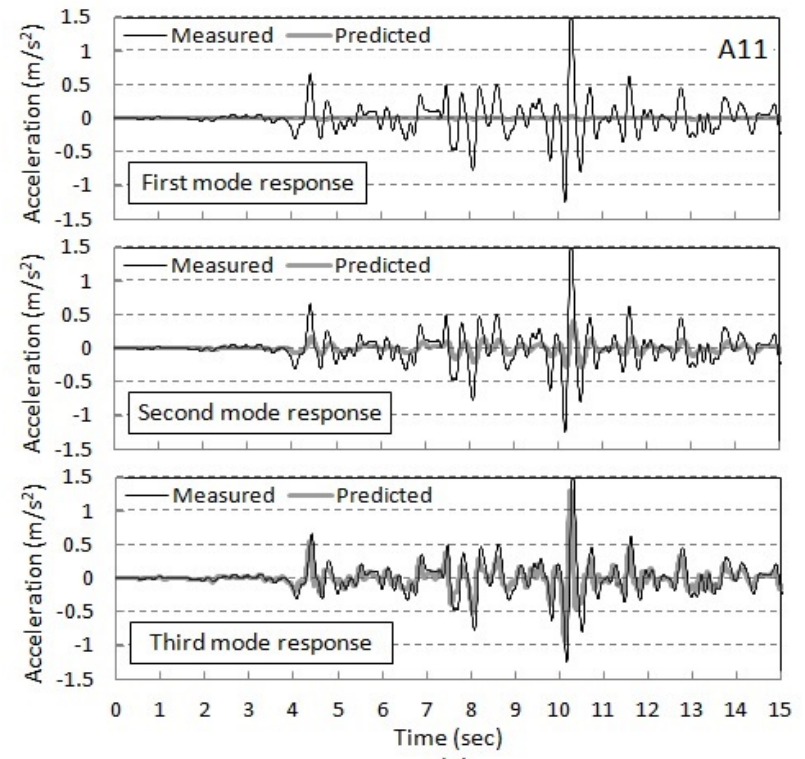

(c)

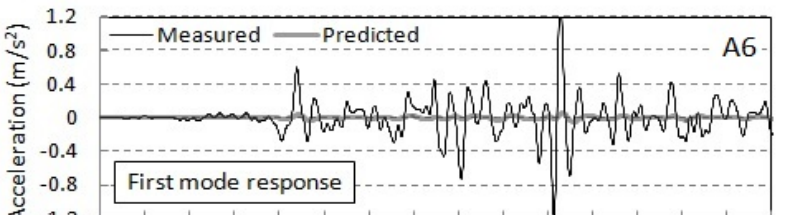

\& -1 .

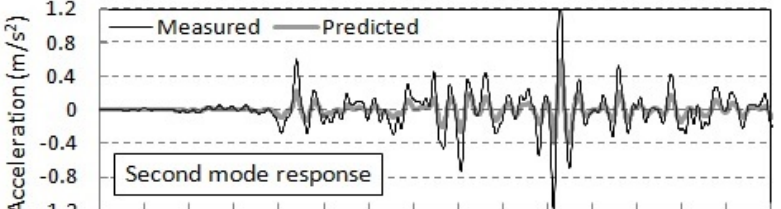

« -1.2
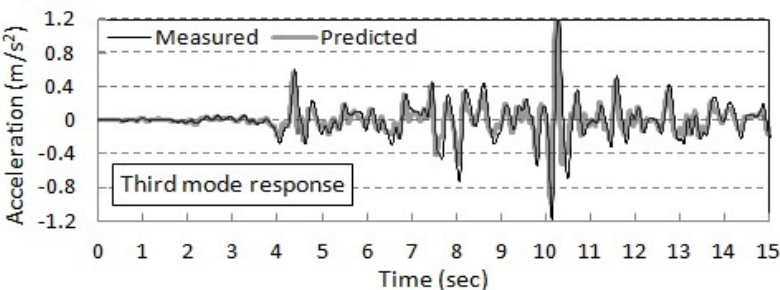

(b)
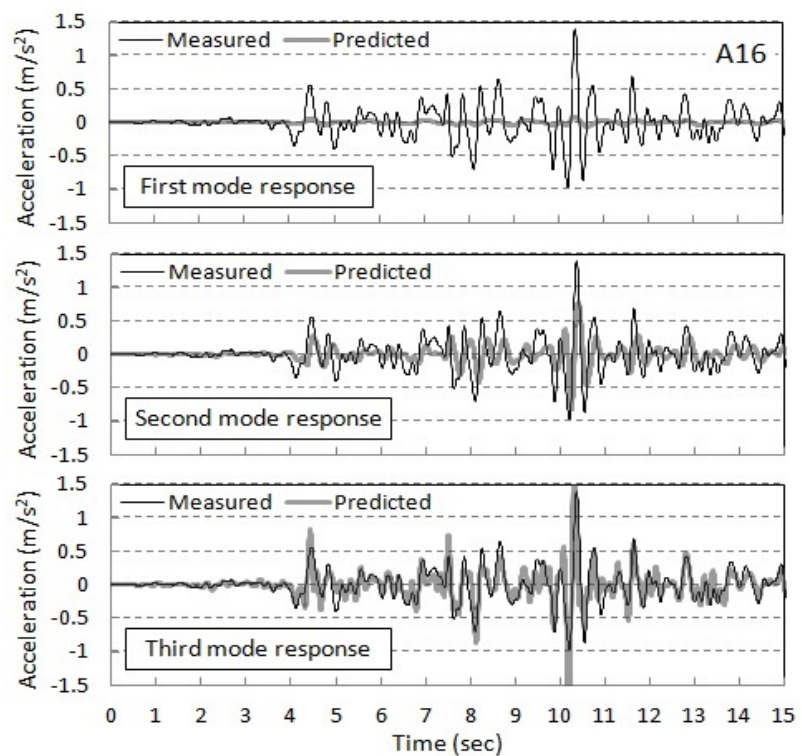

(d)

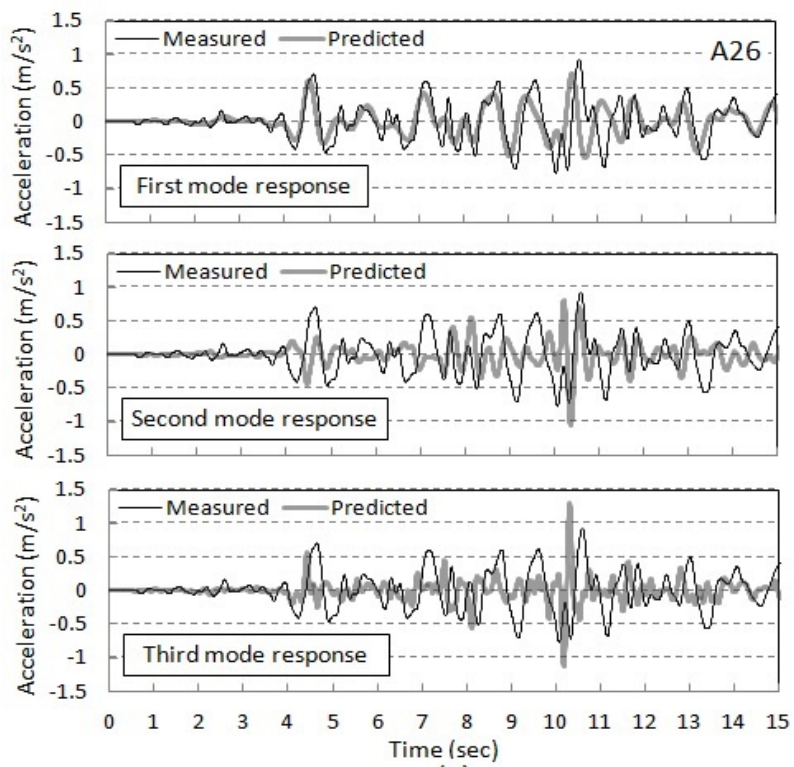

(e)

Fig. 10. The responses of individual modes in the first event (a) A2, (b) A6, (c) A11, (d) A16, (e) A26 


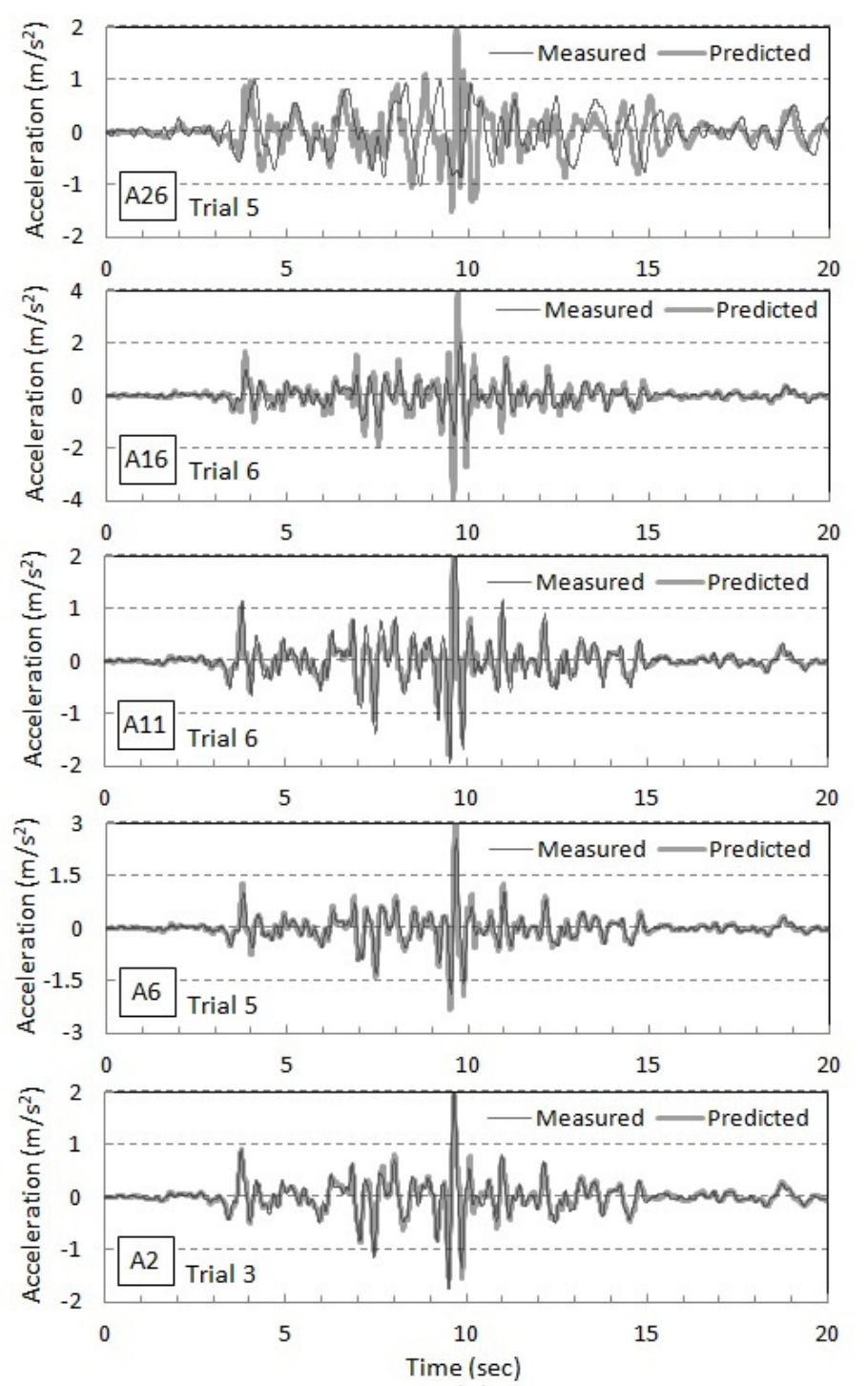

(a)
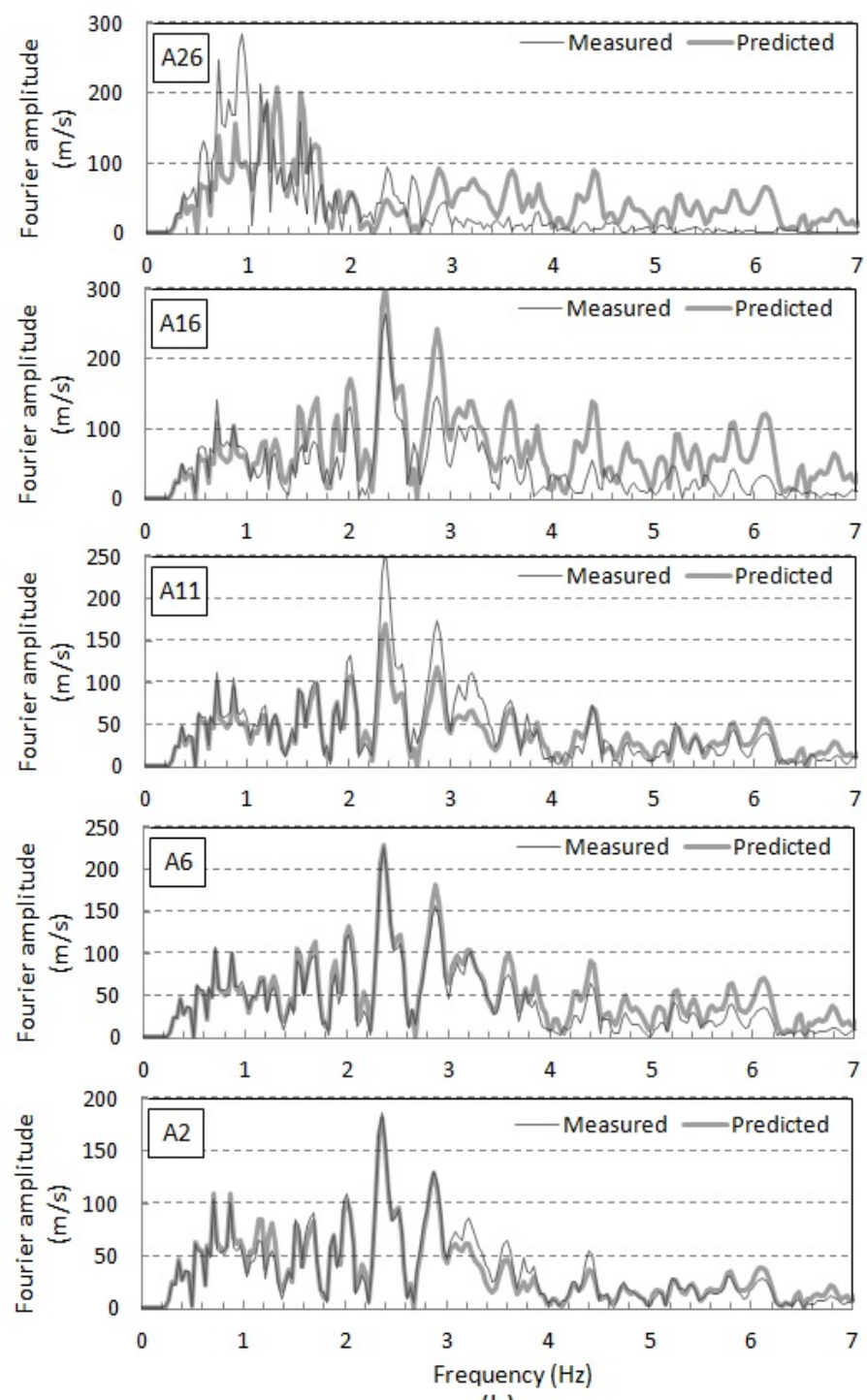

(b)

Fig. 11. Response predictions for the second event (a) time histories (b) Fourier amplitude spectra 

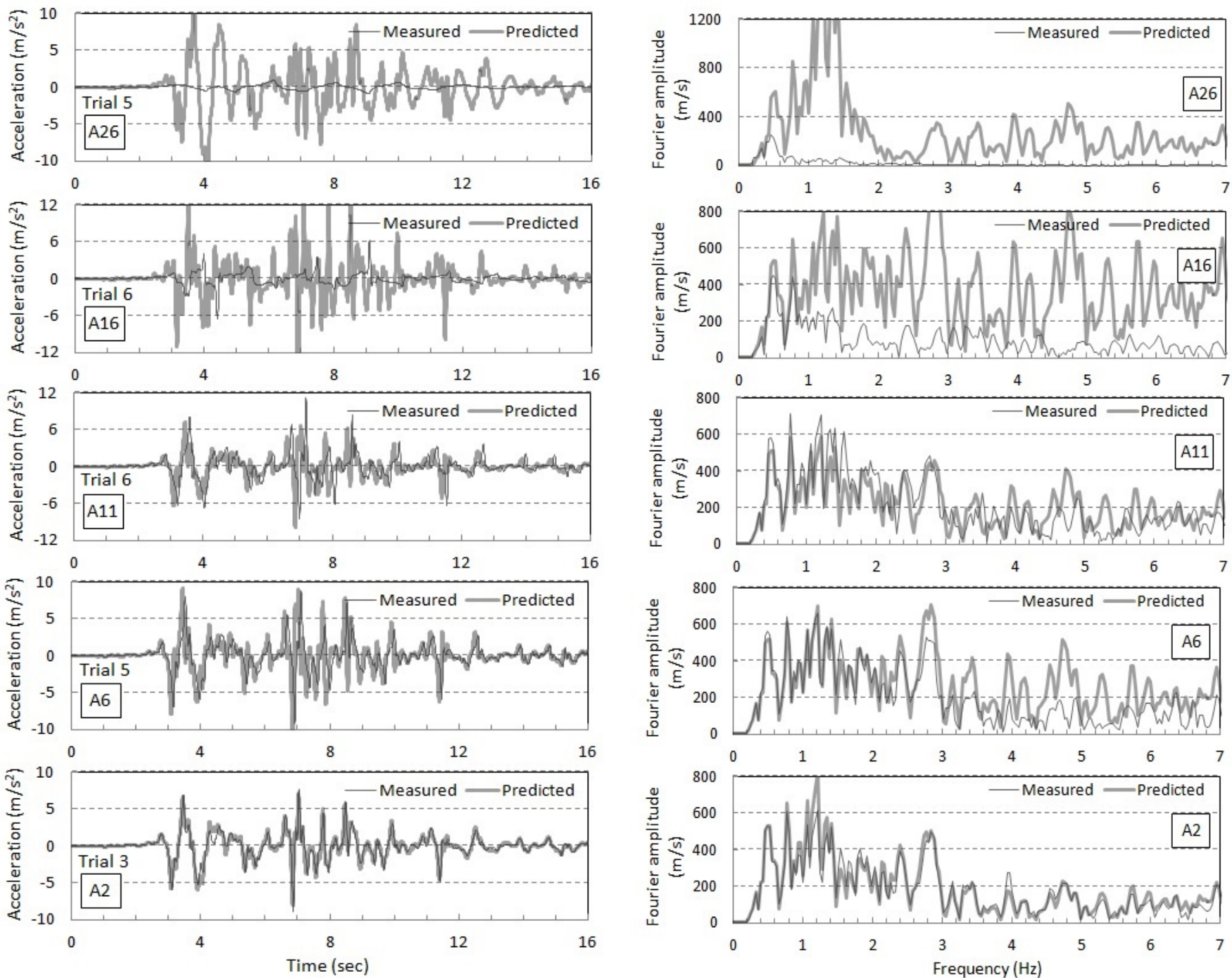

(a)

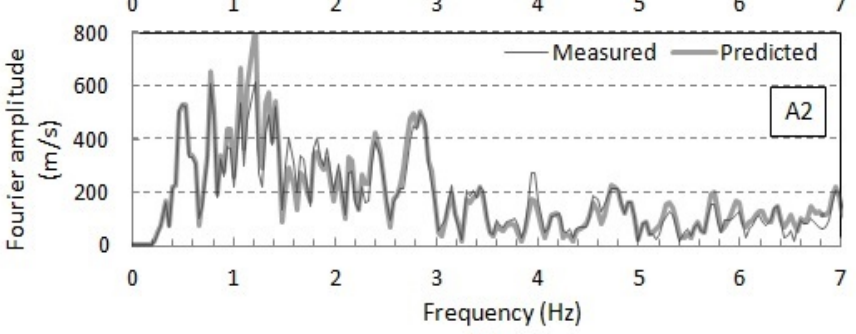

(b)

Fig. 12. Response prediction for the third event (a) time histories (b) Fourier amplitude spectra 